This item was submitted to Loughborough's Research Repository by the author.

Items in Figshare are protected by copyright, with all rights reserved, unless otherwise indicated.

\title{
Reliability, risk and lifetime distributions as performance indicators for life- cycle maintenance of deteriorating structures
}

PLEASE CITE THE PUBLISHED VERSION

http://dx.doi.org/10.1016/j.ress.2013.09.013

\section{PUBLISHER}

(C) Elsevier

\section{VERSION}

AM (Accepted Manuscript)

\section{PUBLISHER STATEMENT}

This work is made available according to the conditions of the Creative Commons Attribution-NonCommercialNoDerivatives 4.0 International (CC BY-NC-ND 4.0) licence. Full details of this licence are available at: https://creativecommons.org/licenses/by-nc-nd/4.0/

\section{LICENCE}

CC BY-NC-ND 4.0

\section{REPOSITORY RECORD}

Barone, Giorgio, and Dan M. Frangopol. 2019. "Reliability, Risk and Lifetime Distributions as Performance Indicators for Life-cycle Maintenance of Deteriorating Structures". figshare. https://hdl.handle.net/2134/16761. 


\title{
Reliability, risk and lifetime distributions as performance indicators for life-cycle maintenance of deteriorating structures
}

\author{
Giorgio Barone ${ }^{1}$ and Dan M. Frangopol ${ }^{2 *}$
}

\begin{abstract}
Structural capacity deterioration is among the main causes of increasing failure probabilities of structural systems, thus maintenance interventions are a crucial task for their rational management. Several probabilistic approaches have been proposed during the last decades for the determination of cost-effective maintenance strategies based on selected performance indicators. However, benefits and drawbacks of each performance indicator with respect to the others should be further analyzed. The objective of this paper is to investigate probabilistic approaches based on the annual reliability index, annual risk, and lifetime distributions for life-cycle maintenance of structural systems. Maintenance schedules are obtained for representative series, parallel, and series-parallel systems considering total restoration of component resistances whenever a prescribed threshold, based on a selected performance indicator, is reached. Effects related to different structural configurations and correlation among failure modes are investigated. The superstructure of an existing bridge is used to illustrate the presented approaches.
\end{abstract}

Author keywords: reliability; risk; lifetime distribution; life-cycle maintenance; deteriorating structures.

\footnotetext{
${ }^{1}$ Research Associate, Department of Civil and Environmental Engineering, Engineering Research Center for Advanced Technology for Large Structural Systems (ATLSS Center), Lehigh University, 117 ATLSS Dr., Bethlehem, PA 18015-4729, USA, gib212@lehigh.edu.

${ }^{2}$ Professor and the Fazlur R. Khan Endowed Chair of Structural Engineering and Architecture, Department of Civil and Environmental Engineering, Engineering Research Center for Advanced Technology for Large Structural Systems (ATLSS Center), Lehigh University, 117 ATLSS Dr., Bethlehem, PA 18015-4729, USA, dan.frangopol@lehigh.edu, *Corresponding Author.
} 


\section{Introduction}

The economic growth of developed countries has led to the creation of extended civil infrastructure networks and facilities. During their life-cycle, these structural systems have been often subjected to natural hazards and aging phenomena caused by environmental and mechanical stressors that decrease their initial performance. At the same time, the current social and political trends promote maintaining existing structures for extended periods of time due to the high direct and indirect costs associated with their eventual replacement. Moreover, several of these structures have significantly lower structural performance today than was initially associated with their designs. This is due to the increasing demand during their operating conditions, and the often ineffective or inappropriate maintenance [1].

Accurate modeling of structures, hazards, stressors and load effects is a major challenge for the structural engineering community. Because of several uncertainties related not only to the structural models, but also to randomness inherent within natural phenomena and loads, probabilistic methods provide the most rational way to obtain high accuracy predictive models, aiming at making optimal decisions for maintenance of the structures. Remarkable contributions have been made to the development of techniques for performance and risk assessment during the structural life-cycle of both individual structures and networks, in the effort of achieving a comprehensive integrated framework and ensuring adequate structural reliability through optimization techniques [2]. Risk assessment and subsequent decision making has been recognized, in recent years, as being of the utmost importance [3-5]. In this context, a comprehensive guideline on risk-based decision making, including system and network modeling, hazard analysis, risk quantification by risk indicators and risk reduction measures, has been proposed [6]. Indicators for assessing the time-dependent performance of damaged bridges, in terms of structural vulnerability, redundancy, and robustness, have been also investigated [7].

The objective of this paper is to investigate probabilistic approaches based on annual reliability index, annual risk, and lifetime distributions for life-cycle maintenance of structural systems. In particular, different approaches to the problem of determining 
maintenance schedules are discussed. The aim of this paper is to provide indications on advantages and drawbacks of these indicators. Threshold-based maintenance problems are solved by analyzing the effects of various system configurations and failure mode correlations. Maintenance options are restricted to essential maintenance, implying total restoration of component resistance after repair. It is assumed that, when a prescribed threshold is reached, maintenance is performed.

This paper is composed of two main parts. In the first one, two point-in-time performance indicators are introduced, the annual reliability index and annual risk, and are used for solving maintenance problems. The annual reliability index has been proposed as an important tool for the assessment of optimal maintenance plans in civil $[8,9]$ and marine structures [10, 11]. Single objective optimization [12] and multi-objective optimization techniques [13] involving reliability analysis and cost-based decision making have been reported. While the reliability index is uniquely connected to the failure probability of the structure, risk takes into account direct and indirect consequences associated with total or partial failure of the system and it is defined as the product of failure probability and consequences estimated in monetary terms [14]. Risk analysis gives maintenance priority to those components having the worst consequences, not only economically, but also socially and environmentally.

In the second part of this paper, lifetime distribution approaches are discussed. Representation of structural performance through lifetime distributions has the advantage that it can be used via closed-form expressions. On the other hand, a lifetime distribution represents the overall effect of all variables involved in the structural resistance and loads, therefore the effect of a single random variable is not easy to find. A further limitation when dealing with system analysis is that closed-form solutions are usually known only for the two particular cases of statistically independent and perfectly correlated failure modes. Maintenance strategies based on lifetime distributions may take into considerations importance factors, giving indications on which components should be repaired to obtain the most beneficial effect on the entire system [15]. The main advantage of using lifetime distributions is their computational efficiency, making them particularly suitable for 
optimization methods [16]. Herein, threshold-based essential maintenance is examined with respect to availability and hazard functions. Finally, the considered approaches are applied to the superstructure of an existing bridge.

\section{Maintenance for improving life-cycle performance}

Several strategies can be considered for improving the life-cycle performance of a system. Maintenance interventions have, in general, two different aims: (a) blocking or slowing down the structural deterioration process, therefore increasing the time required to reach a predefined limit state; or (b) restoring, partially or totally, the resistance of one or more components of the structure when a given condition is reached, to improve the performance of the system. The first strategy is usually categorized as preventive maintenance; in general, preventive maintenance is applied at prescribed time instants during the lifetime of the system. The second one, namely essential maintenance, is instead usually performed when one or more performance indicators reach predefined thresholds, representative of degrading states of the structure. In this paper, the latter approach is considered. Figure 1 shows the effect of essential maintenance by assuming that the structural resistance is returned to its initial value after repair. Time delay for performing repair is not considered; therefore, the resistance is instantaneously increased at the repair time, and the cost of the maintenance intervention is concentrated at the same instant. It has to be noted that the load effect is, in general, increasing over time due, for example, to the increasing demand in terms of traffic load to which bridges are usually subjected. Such load is not affected by maintenance actions during the structural life-cycle.

Repair priority can be given to one or more structural components, based on their effects on the system failure and possible consequences. The decision-making process may lead to different repair choices and different maintenance times depending on which performance indicator is considered. In the following, different configurations of a threecomponent system are analyzed, and essential maintenance schedules are evaluated with respect to different performance indicators and thresholds. In general, structural systems can be modeled as either series, parallel, or series-parallel systems. Herein, these three different 
configurations will be analyzed, considering for each one of them two cases: (a) statistical independence, and (b) perfect correlation between their failure modes. These cases are associated with lower and upper bounds of the system failure probability.

\section{Annual reliability and annual risk as performance indicators}

A rational way to treat uncertainties arising from natural randomness, modeling, and prediction imperfections is to consider probabilistic approaches. In this context, failure probability of a system is defined as the probability of violating one or more limit states associated with the system failure modes. The performance function $g(t)$ for a given failure mode is generally defined as:

$$
g(t)=r(t)-q(t)
$$

where $r(t)$ and $q(t)$ are the instantaneous resistance and load effect at the time instant $t$, respectively. Resistance and load are time-dependent random variables; for engineering systems, if no maintenance is considered, resistance is usually deteriorating over time, while loads are increasing. Considering a system with several failure modes, the point-in-time system failure probability $P_{\text {sys }}$ at time $t$ can be evaluated as:

$$
P_{\text {sys }}(t)=P\left[\text { any } g_{i}(t)<0\right] \quad \forall t>0
$$

where $g_{i}(t)$ is the performance function associated with the $i$-th system failure mode.

Determining the system failure probability is usually a formidable task, requiring solution of multiple integrals whose dimension increases with the number of failure modes. For this reasons, various approximation methods have been proposed. The most used approximate methods for obtaining the probabilities of occurrence of various failure modes are first-order reliability method (FORM) and second-order reliability method (SORM) that allow to solve the problem by approximating the limit state surface in the standardized normal space, at the most likely failure point, with a linear function and a second order 
surface, respectively. Given the definition of system failure state in Eq. (2), the associated reliability index $\beta(t)$ can be determined in approximate form as:

$$
\beta(t)=\Phi^{-1}\left(1-P_{\text {sys }}(t)\right)
$$

where $\Phi$ is the standard normal cumulative distribution function. In usual applications, the probability of failure and the associated reliability index are evaluated at constant time intervals. Herein, a one year time interval is used, and reference will be made to the annual reliability index.

During recent years, due to the increasing necessity of taking into account consequences associated with partial and/or total system failure, risk-based decision making has become an important tool for maintenance optimization. Risk assessment may be either qualitative or quantitative. Qualitative risk assessment deals, in general, with simple descriptions of the types of hazards, their consequences and likelihood, reporting all these aspects in opportunely built risk matrices [17]. Herein, quantitative risk assessment is considered; risk associated with a component failure is defined as the product of the $i$-th component failure probability $P_{i}(t)$ at time $t$ and the consequences $C_{i}(t)$ associated with its failure:

$$
R_{i}(t)=P_{i}(t) C_{i}(t)
$$

The most common way of quantifying consequences is to determine the various losses associated with failure and their effective (or equivalent) cost. Herein, the cost associated with consequences of component/system failure is composed of the direct cost $C^{\text {dir }}$ and the indirect cost $C^{\text {ind }}$. The first type refers to monetary loss deriving from component failure, and can be estimated by the rebuilding or replacement costs of the failed components. Indirect consequences are more difficult to quantify, since they are dependent on the subsequent system failure and its consequences in a broader perspective, taking into account different aspects not only strictly economic, but also, for example, safety and environmental loss. 
Safety loss indicates a measure of damage inflicted to persons due to the system failure, in terms of fatalities, injuries or generic health issues. Environmental loss indicates, instead, degradation or contamination of the environment subsequent to the system failure [18]. Quantification of indirect consequences can be cumbersome when the system is part of a network, since the interaction with other systems in the same network has to be analyzed. Also, consequences associated with safety or environmental aspects are hardly quantifiable in monetary terms (for example, the monetary value of human life).

Analogously, direct and indirect risk can be evaluated. In particular, considering failure of a single component $i$, the associated direct and indirect risk can be quantified, respectively, as follows:

$$
\begin{aligned}
& R_{i}^{\text {dir }}(t)=P\left(F_{i} \bar{F}_{j \neq i}\right) C_{i}^{\text {dir }}(t) \\
& R_{i}^{\text {ind }}(t)=P\left(F_{i} \bar{F}_{j \neq i}\right) P\left(F_{\text {sys }} \mid F_{i} \bar{F}_{j \neq i}\right) C_{i}^{\text {ind }}(t)
\end{aligned}
$$

in which $P\left(F_{i} \bar{F}_{j \neq i}\right)$ represents the probability of failure of the $i$-th component at time $t$ when all other components are surviving, and $P\left(F_{s y s} \mid F_{i} \bar{F}_{j \neq i}\right)$ represents the system failure probability conditioned by the event $F_{i} \bar{F}_{j \neq i}$. Therefore, the total risk associated with the component failure is obtained as:

$$
R_{i}^{\text {tot }}(t)=R_{i}^{\text {dir }}(t)+R_{i}^{\text {ind }}(t)
$$

The total risk associated with the whole system is evaluated, in analogous way, as:

$$
R_{\text {sys }}^{\text {tot }}(t)=P_{\text {sys }}(t)\left(C^{\text {dir }}+C^{\text {ind }}\right)
$$

where $P_{\text {sys }}(t)$ is the failure probability of the whole system, and $C^{\text {dir }}$ and $C^{\text {ind }}$ are direct and indirect consequences associated with the whole system failure. To propose a direct comparison with the annual reliability index, herein annual risk has been used. In practical applications, risk associated with the remaining structural life-cycle is to be used. While in real applications the life-time risk should be applied, according to theoretical decision theory 
and risk analysis, it would not allow a straightforward comparison with the annual reliability index. Therefore, in the following, annual risk is used to allow this comparison.

For illustrative purposes, the four systems shown in Figure 2 are considered. These systems consist of the same three components, arranged in series, parallel and series-parallel and are governed by the performance function in Eq. (1). For the series-parallel case, two different arrangements are considered (see Figure 2(c) and 2(d)). It is assumed that the resistance of the components is deteriorating, due to a deterministic progressive reduction of their cross-sectional areas over time [19]. Components resistances are considered lognormal random variables, with mean $\mu_{r_{i}}(t)$ and standard deviation $\sigma_{r_{i}}(t)$ :

$$
\begin{aligned}
& \mu_{r_{i}}(t)=\left(1-D_{i}\right) A_{i}(t-1) \mu_{F_{y}}=\left(1-D_{i}\right)^{t} A_{0 i} \mu_{F_{y}} \\
& \sigma_{r_{i}}(t)=\left(1+D_{i}\right)^{t} A_{0 i} \sigma_{F_{y}}
\end{aligned}
$$

where $A_{0 i}=$ initial cross-sectional area of the $i$-th component (3.0, 3.1 and $2.9 \mathrm{~cm}^{2}$ for components one, two and three, respectively), $D_{i}=$ deterioration rate (0.003 per year for components one and two, 0.001 per year for component three), while $\mu_{F_{y}}$ and $\sigma_{F_{y}}$ are mean and standard deviation of the yield strength (250 MPa and $10 \mathrm{MPa}$, respectively, for all components).

Analogously, the time-variant load effect is considered lognormal distributed with mean and standard deviation, respectively, as:

$$
\begin{aligned}
& \mu_{q_{i}}(t)=\left(1-l_{i}\right)^{t} \mu_{q_{0 i}} \\
& \sigma_{q_{i}}(t)=0.05 \mu_{q_{i}}(t)
\end{aligned}
$$

where $l_{i}=$ rate of increase of load over time and $\mu_{q_{0 i}}=$ mean load at the initial time (i.e. 0.0002 per year and $55 \mathrm{kN}$, respectively, for all components). Two different correlation cases have been studied. In the first case, failure mode of the components have been considered statistically independent, and therefore having no correlation (i.e., $\rho=0$, where $\rho=$ correlation coefficient). In the second case, perfect correlation ( $\rho=1)$ has been considered. 
The analysis is herein limited to these two correlation conditions; however, in practical applications, correlation between failure modes should be properly modeled and, in some cases, sensitivity analysis with respect to the various parameters involved in the calculation may be necessary.

Annual failure probabilities and annual reliability indices for the three components and systems in Figure 2 have been evaluated, considering 50 years of service life, using the software RELSYS (RELiability of SYStems) [20]. The software uses FORM to first compute the failure probability of all components of the system; then, it progressively reduces the system to equivalent subsystems until a single equivalent component remains. For the four systems in Figure 2, annual risks associated with both system and component failures have been evaluated. Indirect consequences associated with the system failure have been assigned the overall nominal value $10^{6}$. Direct consequences, $C_{i}^{\text {dir }}$, associated with each component (nominal replacement/repair cost of each component) have been assumed as $10^{4}$ for components one and two, and $10^{5}$ for component three). Annual risk associated with failure of each component has been calculated using Eqs. (5) and (6). As shown in Eq. (5), two different probabilities must be determined. The first one is the probability of failure of the $i$-th component when the other two components $j$ and $k$ survive. For the statistical independent and perfectly correlated cases the probability of this event is computed, respectively, as:

$$
\begin{aligned}
& P\left(F_{i} \bar{F}_{j \neq i}\right)_{\rho=0}=P\left(F_{i} \bar{F}_{j} \bar{F}_{k}\right)_{\rho=0}=P\left(F_{i}\right)\left(1-P\left(F_{j}\right)\right)\left(1-P\left(F_{k}\right)\right) \\
& P\left(F_{i} \bar{F}_{j \neq i}\right)_{\rho=1}=P\left(F_{i} \bar{F}_{j} \bar{F}_{k}\right)_{\rho=1}=\max \left\{0 ; \quad P\left(F_{i}\right)-\max \left[P\left(F_{j}\right) ; \quad P\left(F_{k}\right)\right]\right\}
\end{aligned}
$$

The probability of failure of the system conditioned by failure of only $i$-th component, $P\left(F_{\text {sys }} \mid F_{i} \bar{F}_{j \neq i}\right)$, can be evaluated as the failure probability of a new reduced system in which the $i$-th component has been removed.

Figure 3 shows the annual failure probabilities and the annual reliability index for the three-component systems illustrated in Figure 2. For the same systems, annual risk is reported in Figures 4 and 5 for statistically independent and perfectly correlated failure modes, respectively. For the latter case, by substituting Eq. (10) into Eq. (5), at each instant the 
annual risk associated with a single component has to be zero for all components except one. As expected, the highest annual failure probability (i.e., lowest annual reliability index) is obtained for the series system A in the statistical independent case, while the lowest annual failure probability (i.e., highest annual reliability index) and annual risk are obtained for the parallel configuration B in the statistical independent case (see Figure 3b). In particular, the latter differs in several orders of magnitude in terms of both annual failure probability and annual risk compared to all other systems.

\section{Reliability-based and risk-based maintenance}

Since the aim of this paper is to examine how different performance indicators affect decision-making for maintenance strategies, in this section maintenance scheduling is determined based on system thresholds defined in terms of annual reliability index and annual risk. Two different strategies have been considered for the two performance indicators.

Firstly, reliability-based maintenance has been analyzed. Two arbitrary system reliability thresholds, $\beta_{t, 1}=3.5$ and $\beta_{t, 2}=4.0$, have been selected. For each case, maintenance is performed whenever the predefined threshold $\beta_{t, k}$ is reached, within 50 years of service life. Actually, the threshold will be reached at a point-in-time within a one year interval; however, maintenance is performed at the beginning of the following year, when the annual system reliability index is, actually, lower than the predefined threshold. At such instant in time, essential maintenance is performed on the component with the lowest annual reliability. This approach disregards failure consequences.

The second investigated strategy is risk-based maintenance. In this case, maintenance is performed on the component having the highest associated annual risk, when the system risk-threshold $R_{t, k}$ is exceeded. Therefore, direct and indirect consequences associated with each component failure assume a relevant weight in the decision process. If the loss associated with failure of a specific component is much higher than the loss associated with any other component, then it has to be expected that the highest repair priority will be given to this component. Two system thresholds $R_{t, k}$ have been selected, so that they are associated 
with the same system annual failure probability (i.e. the same corresponding reliability thresholds $\beta_{t, k}$ ). Therefore:

$$
R_{t, k}=P_{s y s, k}\left(C^{\text {dir }}+C^{\text {ind }}\right)=\left(\Phi\left(-\beta_{t, k}\right)\right)\left(C^{\text {dir }}+C^{\text {ind }}\right)
$$

By substituting values of $\beta_{t, 1}$ and $\beta_{t, 2}$ into Eq. (11), the risk thresholds $R_{t, 1}=261$ and $R_{t, 2}=35$ have been obtained. For both strategies, statistically independent and perfectly correlated failure modes have been considered.

When failure modes are statistically independent, both the reliability and the risk thresholds are never reached for the parallel system B in the considered 50 years of service life, due to its extremely low annual failure probability. For the other cases, since the reliability and risk thresholds are related through Eq. (11), the first maintenance time occurs at the same instant when using $\beta_{t, 1}$ or $R_{t, 1}$ (or analogously, when using $\beta_{t, 2}$ or $R_{t, 2}$ ) for all systems. On the other hand, the component with lowest annual reliability index may be different from the one with highest annual risk. Following the reliability-based approach, in several cases repair actions on single components are associated with very low improvement of the system reliability. This happens because the same importance is given to all components.

Conversely, in the risk-based approach, component importance is based on failure consequences. This consequence-based importance is mainly dependent on the component location inside the considered system whenever the indirect consequences are much higher than the direct ones, since, in this case, system failure consequences are much more significant than the replacement cost of the component itself (direct consequences). Thus, in the proposed examples, in which indirect consequences are assumed one order of magnitude higher than the most expensive component replacement cost, the same maintenance plans are obtained for the series system A using either reliability or risk-based approach. In contrast, in the two series-parallel systems $C$ and $D$, the number of repair actions is lower in the riskbased maintenance case (i.e., total cost of the maintenance plan is lower). Moreover, comparing systems $\mathrm{C}$ and $\mathrm{D}$, it can be noticed that, for the risk case, repair priority is always 
given to the series component 3 in Figure 2, even if this is not the most expensive one (e.g., in system D).

When perfectly correlated failure modes are considered, repair is performed at least once for all systems and thresholds. In this case, results obtained considering reliability-based maintenance always match those obtained by a risk-based approach. In fact, when failure modes are perfectly correlated, the component with highest associated annual risk is also the one with the highest annual failure probability, and therefore with the lowest annual reliability. Since reliability and risk thresholds have been selected to correspond to the same system annual failure probability (Eq. (11)), then matching risk and reliability thresholds ( $\beta_{t, 1}$ and $R_{t, 1} ; \beta_{t, 2}$ and $R_{t, 2}$ ) will be reached at the same instant of time, thus, the same components will be repaired.

Maintenance schedules obtained for the four systems in Figure 2, considering reliability or risk thresholds, are graphically represented in Figures 6 and 7 for statistically independent and perfectly correlated failure modes, respectively. Detailed annual reliability and annual risk profiles during 50 years of service life are illustrated for the series-parallel system C in Figure 8 for the case of reliability-based maintenance considering the threshold $\beta_{t, 1}$ and in Figure 9 for risk-based maintenance considering the threshold $R_{t, 1}$. Whenever two or more repair actions are required in a one year interval, it is considered that they are both performed at the same time (i.e., when the first repair is required). Figures 8(a) and (b) show annual reliability and annual risk profiles, respectively, when the threshold $\beta_{t, 1}$ is used under the assumption of statistically independent failure modes, while Figures 8(c) and (d) are associated with the case of perfectly correlated failure modes. Annual risk and annual reliability profiles, respectively, corresponding to the risk threshold $R_{t, 1}$ are plotted in Figures 9(a) and (b) for statistically independent failure modes, and Figures 9(c) and (d) for the perfectly correlated case.

Cumulative cost profiles of all the considered maintenance plans are shown in Figure 10 for systems A, C, and D. The parallel system B has not been shown, since only two possible results are obtained, with only one or two repairs. While for the series system A the most expensive plans are associated with the cases of statistically independent failure modes, 
for the series-parallel systems $C$ and $D$ the most expensive plan has the same cost for both correlation cases. Regarding the series-parallel systems, as mentioned previously, the risk approach allows a reduction of the total maintenance cost by reducing the number of required repair actions.

\section{Survivor, availability and hazard functions}

A disadvantage of the previously examined performance indicators is the lack of closed-form solutions over time. Annual reliability index and annual risk have to be evaluated at predetermined instants of time, returning a solution that, not only is not continuous in time, but usually requires approximate numerical methods that may be computational inefficient for complex system analysis. Lifetime distributions are alternative representations of the lifetime structural reliability through continuous functions that can be defined in closed-form by considering the time to failure of components and systems as a random variable [21]. On the other hand, considering directly the performance functions associated with the failure mode of the systems provides a clear understanding of the separate effect of the contribution of each random variable to either resistance or loads. Instead, lifetime distributions represent the combined effect of all the uncertainties on the structure, lacking an explicit distinction between resistance and load effects. A second disadvantage of using lifetime distributions is due to the fact that closed-form solutions are usually known only for statistically independence and perfect correlation among system components, while, using annual reliability or annual risk allows to take into account any possible degree of correlation among the random variables involved in the definition of the system performance functions.

Among the different known lifetime distributions, the more appropriate ones for threshold-based approaches are availability and hazard functions. It is convenient first to introduce the survivor function, representing the probability that a component or system is still surviving (not failed) at a given time instant:

$$
S(t)=P\left[T_{F} \geq t\right]
$$


where $T_{F}=$ time to failure of the considered component or system [22]. The availability function $A(t)$ is, analogously, the probability of the system still functioning at a given time instant, and it is equivalent to the survivor function for non-repairable systems [21]. While the survivor function is a non-increasing function over time, the availability function can increase its value when a repair is performed; however, it is bounded in the interval $[0,1]$.

The hazard function is defined as the limit for $\Delta t \rightarrow 0$ of the failure probability in the time interval $[t, t+\Delta t]$, conditioned by the system still surviving at time $t$, and averaged over the same time interval $\Delta t$, that is:

$$
h(t)=\lim _{\Delta t \rightarrow 0} \frac{P\left[t \leq T_{f} \leq t+\Delta t \mid T_{f} \geq t\right]}{\Delta t}=-\frac{S^{\prime}(t)}{S(t)}
$$

where $S^{\prime}(t)$ is the derivative of the survivor function over time. Therefore, while the availability (and the survivor function) is directly related to the probability of failure of the system, the hazard function gives an indication of the rate of system failure.

For the systems A, B, and C in Figure 2, the closed-form of the system survivor (and equivalently availability) function and hazard functions can be obtained for both the statistically independent and perfectly correlated cases using, for example, the minimal path or minimal cut set techniques [22]. In particular, denoting $S_{S}(t), S_{P}(t)$, and $S_{S P}(t)$ the survivor functions and $A_{S}(t), A_{P}(t)$, and $A_{S P}(t)$ the availability functions for series, parallel and series-parallel systems, respectively, and $S_{i}(t)$ the survivor functions of the single components, the following relations, valid for statistically independent and perfectly correlation among components, are valid:

$$
\begin{aligned}
& S_{S}(t)_{\rho=0}=A_{S}(t)_{\rho=0}=\prod_{i=1}^{3} S_{i}(t) \\
& S_{S}(t)_{\rho=1}=A_{S}(t)_{\rho=1}=1-\max \left(1-S_{i}(t)\right)
\end{aligned}
$$




$$
\begin{aligned}
& S_{P}(t)_{\rho=0}=A_{P}(t)_{\rho=0}=1-\prod_{i=1}^{3}\left(1-S_{i}(t)\right) \\
& S_{P}(t)_{\rho=1}=A_{P}(t)_{\rho=1}=1-\min \left(1-S_{i}(t)\right) \\
& S_{S P}(t)_{\rho=0}=A_{S P}(t)_{\rho=0}=\left(1-\prod_{i=1}^{2}\left(1-S_{i}(t)\right)\right) S_{3}(t) \\
& S_{S P}(t)_{\rho=1}=A_{S P}(t)_{\rho=1}=1-\max \left\{\min \left[\left(1-S_{1}(t)\right) ; \quad\left(1-S_{2}(t)\right)\right] ; \quad\left(1-S_{3}(t)\right)\right\}
\end{aligned}
$$

The hazard functions for the same systems can be straightforwardly evaluated by inserting Eqs. (14), (15), and (16) into Eq. (13), thus obtaining the following equations:

$$
\begin{aligned}
& h_{S}(t)_{\rho=0}=\sum_{i=1}^{3} h_{i}(t) \\
& h_{S}(t)_{\rho=1}=\sum_{i=1}^{3} h_{i}(t) \alpha_{i}(t) \\
& h_{P}(t)_{\rho=0}=\frac{\sum_{i=1}^{3} h_{i}(t) S_{i}(t) \prod_{\substack{j=1 \\
j \neq i}}^{3}\left(1-S_{j}(t)\right)}{1-\prod_{i=1}^{3}\left(1-S_{i}(t)\right)} \\
& h_{P}(t)_{\rho=1}=\sum_{i=1}^{3} h_{i}(t) \beta_{i}(t) \\
& h_{S P}(t)_{\rho=0}=\frac{h_{1}(t) S_{1}(t)\left(1-S_{2}(t)\right)+h_{2}(t) S_{2}(t)\left(1-S_{1}(t)\right)}{S_{1}(t)+S_{2}(t)-S_{1}(t) S_{2}(t)}+h_{3}(t) \\
& h_{S P}(t)_{\rho=1}=\sum_{i=1}^{3} h_{i}(t) \gamma_{i}(t)
\end{aligned}
$$

where coefficients $\alpha_{i}(t), \beta_{i}(t)$, and $\gamma_{i}(t)$ are defined as:

$$
\alpha_{i}(t)= \begin{cases}1 & \text { if } 1-S_{i}(t)=\max _{j}\left(1-S_{j}(t)\right) \quad i, j=1,2,3 \\ 0 & \text { otherwise }\end{cases}
$$




$$
\begin{gathered}
\beta_{i}(t)= \begin{cases}1 & \text { if } 1-S_{i}(t)=\min _{j}\left(1-S_{j}(t)\right) \quad i, j=1,2,3 \\
0 & \text { otherwise }\end{cases} \\
\gamma_{i}(t)= \begin{cases}1 & \text { if } 1-S_{i}(t)=\max \left\{\min \left[\left(1-S_{1}(t)\right) ; \quad\left(1-S_{2}(t)\right)\right] ; \quad\left(1-S_{3}(t)\right)\right\} \quad i=1,2,3(22) \\
0 & \text { otherwise }\end{cases}
\end{gathered}
$$

In the following it is assumed that the time to failure of the components in Figure 2 follows the Weibull distribution:

$$
\begin{aligned}
& S_{i}(t)=e^{-\left(\lambda_{i} t\right)^{k_{i}}} \\
& h_{i}(t)=k_{i} \lambda_{i}^{k_{i}} t^{k_{i}-1}
\end{aligned}
$$

considering $\lambda_{1}=6 \times 10^{-3}, k_{1}=2.0$ for the first component, $\lambda_{2}=8 \times 10^{-3}, k_{2}=2.2$ for the second component, and $\lambda_{3}=3 \times 10^{-3}, k_{3}=1.4$ for the third one. Availability and hazard functions for both cases of statistically independent and perfectly correlated failure modes are depicted in Figure 11 for the systems A, B and C.

\section{Availability-based and hazard-based maintenance}

The main advantage of utilizing lifetime distributions for developing efficient maintenance strategies is that it is possible to operate directly in analytical form. Maintaining the previous assumptions that only essential maintenance is applied on the system, after $n$ maintenance actions have been performed at the times $T_{1}, \ldots, T_{n}$ over a component, its survivor function can be expressed as:

$$
S_{i}(t)= \begin{cases}S_{i}(t) & t<T_{1} \\ S_{i}\left(t-T_{n}\right) \prod_{k=1}^{n} S\left(T_{k}-T_{k-1}\right) & T_{n} \leq t<T_{n+1}, k \geq 1\end{cases}
$$


where $T_{0}=0$ is the initial observation time. Eq. (24) properly defines the survivor function as continuous and non-increasing. In other words, the corresponding availability function is obtained by simply restoring the survivor function to its original value after each repair [16]:

$$
A_{i}(t)=S_{i}\left(t-T_{n}\right) \quad T_{n} \leq t<T_{n+1}
$$

In an analogous way, considering the definition of the hazard function Eq. (13), the latter is also restored to its initial value after each repair:

$$
h_{i}(t)=h_{i}\left(t-T_{n}\right) \quad T_{n} \leq t<T_{n+1}
$$

Obviously, Eqs. (24), (25) and (26) refer to each component of the system, while system availability and hazard functions can be determined using minimal path or minimal cut set techniques.

In this section, two different thresholds-based approaches are considered. In the first case, attention has been focused on the availability function and the following thresholds have been selected: $A_{t, 1}=0.94$ and $A_{t, 2}=0.96$. When these thresholds are reached, essential maintenance is performed on a single component, as it has been done for the reliability-based and risk-based approaches in the previous section. Since only integer maintenance times have been considered, the thresholds can be seen as warning states, so that, when reached between two years, essential maintenance is not performed immediately, but on the following year. Since a closed-form is available for system and component availability functions, the following availability importance factor $I_{A, i}$ has been defined for each component:

$$
I_{A, i}(t)=\frac{\partial A_{s y s}(t)}{\partial A_{i}(t)}
$$

where $A_{s y s}(t)$ is the system availability function and $A_{i}(t)$ is the $i$-th component one. This factor may be normalized as: 


$$
I_{A, i}^{*}(t)=\frac{I_{A, i}(t)}{\sum_{i=1}^{m} I_{A, i}(t)}
$$

where $m$ is the total number of components.

The factor $I_{A, i}^{*}$ gives, at each time instant, a measure of how a variation of the availability of the $i$-th component would affect the system availability. Therefore, in the proposed approach, essential maintenance is performed on the component having the highest availability importance factor at the maintenance time, rather than the lowest availability. Note that $I_{A, i}^{*}=1$ implies that the system availability depends only on the $i$-th component. Conversely, $I_{A, i}^{*}=0$ indicates that the $i$-th component do not contribute at all to the variation of the availability of the system, and an eventual repair of only this component would not improve the system performance. The importance factor is clearly dependent on both the system configuration and correlation among components. Figure 12 shows values of importance factors over time for statistically independent components for systems A, B and C. For the series-parallel system $\mathrm{C}$ the component in series has the highest importance factor during the service life of the structure. On the other hand, for series and parallel systems the most important component is changing over time.

Figure 13 summarizes the results for both systems A and C for the statistically independent case, while Figure 14 reports results for the systems A, B and C for the case of perfectly correlated failure modes. When components are statistically independent, the parallel system availability never reaches the selected thresholds during the considered system service life, and therefore no maintenance schedule is reported for this case in Figure 13. Results obtained for the series-parallel system $C$, considering the threshold $A_{t, 1}$, are reported in terms of system and component availability and hazard functions for the case of statistically independent components in Figures 15(a) and (b), respectively, and for the case of perfect correlation in Figures 15(c) and (d).

The second approach that has been followed is the hazard-based one. Maintenance strategies based on the hazard function by using prescribed thresholds and optimization techniques have been proposed in $[23,24]$. Herein, similar to the availability approach, two 
thresholds are defined, this time in terms of hazard function values: $h_{t, 1}=2 \cdot 10^{-3}$ (years $^{-1}$ ) and $h_{t, 2}=1.5 \cdot 10^{-3}$ (years $\left.^{-1}\right)$. Moreover, a normalized hazard importance factor is defined as:

$$
I_{h, i}^{*}(t)=\frac{I_{h, i}(t)}{\sum_{i=1}^{m} I_{h, i}(t)}
$$

where:

$$
I_{h, i}(t)=\frac{\partial h_{s y s}(t)}{\partial h_{i}(t)}
$$

representing, at each time instant, the effect on the system hazard of a variation in a single component hazard. In this case, essential maintenance is performed on the component with the highest hazard importance factor. Results obtained following this new approach considering the thresholds $h_{t, 1}$ and $h_{t, 2}$ for both statistically independent and perfect correlated cases are reported in Figures 13 and 14 as well. Moreover, for system C, results are shown in detail in Figure 16 for both statistically independent and perfectly correlated failure modes.

There is not a direct relation between the two sets of thresholds $A_{t, \mathrm{k}}$ and $h_{t, \mathrm{k}}$, as there was for reliability and risk thresholds. The aim of the two methods is, indeed, different. When considering the availability threshold, a limit is imposed on the probability of the system being functional at any time. Taking into account the hazard function allows, instead, to impose a limit to the rate of failure (and not the probability of failure) of the system and, therefore, to control how quickly the system tends to become non-functional. This last method may be more or less conservative, in terms of availability, depending on the system configuration and the correlation among the components. The hazard-based method is, in particular, very conservative with respect to the availability-based one for the series system A with statistically independent components due to its fast increasing failure rate. 
For both cases of availability and hazard-based maintenance, using importance factors reduces the number of repaired components. In the reliability and risk approaches, it may be observed that in several cases multiple components are repaired at once. In the risk case, this happens because the component with highest annual risk may be not the most important with respect to the system failure. Analogously, in the proposed reliability-based approach, neglecting any importance factor leads often to repair of components that did not provide a significant contribution to the system performance. Clearly, importance factors may be defined also for the reliability and risk cases, although no closed-form can be given and, therefore, they may be evaluated only numerically, especially when performance functions are nonlinear expressions involving several random variables with various correlations and distributions. The use of importance factors in the availability and hazard-based procedures entails that only the most critical component is repaired each time, except for a few cases in which repairing only the component with highest importance factor is still not sufficient to increase the availability over the threshold level (or to decrease the hazard function below its threshold). Also, comparing results based on availability and hazard, it can be seen how the definition of two different impact factors may lead to totally different strategies, in which priorities are given to different components, still maintaining an adequate system performance level. For example, for the parallel system B with perfectly correlated components, repair is performed only on component three when considering availability, while component two is the most important one with respect to hazard (see Figure 14).

\section{Case study: a reinforced concrete bridge superstructure}

In the following, the four discussed approaches are applied to the superstructure of a reinforced concrete bridge whose model is based on the data provided in [25] for the E-17HS bridge located over interstate highway in Adams County, Colorado. The end spans are herein considered. The bridge deck is supported by four reinforced concrete T-girders and a cross-section of the bridge is shown in Figure 17(a). The bridge has been modeled as a seriesparallel system (Figure 17(b)), so that failure for this bridge is given by either failure of the deck or two adjacent girders. Detailed information on this bridge can be found in [25, 26]. 
Based on these data, two different limit states have been used for the deck and girders. The most critical failure mode for the deck is associated with its flexural behavior. Considering the capacity moment $M_{R}$ and the load moment $M_{L}$, neglecting the effects of the dead load, the associated performance function can be written as [25]:

$$
g_{\text {deck }}=M_{R}-M_{L}=C_{1} A_{d} f_{y, d} \lambda_{d} \gamma_{d}-C_{2} \frac{A_{d}^{2} f_{y, d}^{2} \gamma_{d}}{f_{c, d}}-C_{3} \lambda_{\text {trk }}
$$

where $A_{d}$ is the cross-sectional area of the deck steel reinforcement, $f_{y, d}$ its yield strength, $\lambda_{d}$ is an uncertainty factor related to the depth of the reinforcement into the deck, $f_{c, d}$ is the compressive strength of the deck concrete, $\gamma_{d}$ is a modeling uncertainty factor and $\lambda_{\text {trk }}$ is a random variable representing the effect of the load due to a HS20 truck. Coefficients $C_{i}$ are deterministic $\left(C_{1}=4.323 \times 10^{-1}, C_{2}=4.085 \times 10^{-3}, C_{3}=5.287\right.$ ). For the girders, the shear failure mode is the most critical one and the performance function associated with such failure mode, neglecting the dead load effects, can be written as [25]:

$$
g_{i}=C_{4} \sqrt{f_{c, g}} \lambda_{d, i} \gamma_{g}+C_{5} A_{g, i} f_{y, g} \lambda_{d, i} \gamma_{g}-V_{t r k} I_{f} D_{f}
$$

where $A_{g, i}$ is the cross-sectional area of the shear reinforcement on the $i$-th concrete girder, $f_{y, g}$ the girder reinforcements yield strength, $\lambda_{d, i}$ is the uncertainty factor related to the depth of the reinforcement into the girder, $f_{c, g}$ is the compressive strength of the girder concrete, $\gamma_{g}$ is a modeling uncertainty factor, $V_{t r k}$ is the shear due to a truck load, $I_{f}$ is the girder impact factor, and $D_{f}$ the distribution factor. Also in this case the coefficients $C_{i}$ are deterministic $\left(C_{4}=30.925, C_{5}=5.093\right)$.

Random variables involved into Eqs. (31) and (32) are considered lognormal with means and standard deviations reported in Table 1. Cross-sectional area of the reinforcement bars of both deck and girders are assumed to be subjected to corrosion and, therefore, their value is decreasing over time. Degradation of the reinforcement bars can be caused by several factors, like chloride penetration, alkali aggregate reactions, and concrete carbonation among others [27]. Several corrosion models can be found in literature, depending on the considered 
structural system and environmental factors affecting it ([27] - [31]). Herein, it is assumed that the corrosion of the reinforcement rebars is due to the chloride penetration. In this case, the chloride concentration over time $C_{l}(x, t)\left(\mathrm{g} / \mathrm{mm}^{3}\right)$ can be expressed as the following solution of the second Fick's law [28]:

$$
C_{l}(x, t)=C_{l 0}\left[1-\operatorname{erf}\left(\frac{x}{2 \sqrt{D t}}\right)\right]
$$

in which $x(\mathrm{~mm})$ is the depth of the bars from the concrete surface, $C_{l 0}\left(\mathrm{~g} / \mathrm{mm}^{3}\right)$ is the concentration of the diffusing chloride ions on the surface of the concrete, $D$ is the diffusion coefficient (considered constant in time and space) and erf is the error function. Then, the corrosion initiation time distribution for the reinforcement steel has been evaluated by Monte Carlo based on Eq. (33) and considering a critical threshold limit for the chloride concentration. Finally, the residual reinforcement bars diameter over time has been evaluated as:

$$
d_{b a r}(t)=d_{0}-r\left(t-T_{i n i}\right)
$$

where $d_{0}$ is the initial reinforcement bar diameter (26 $\mathrm{mm}$ for the deck, $18 \mathrm{~mm}$ for the shear reinforcement of the girders); $r$ and $T_{i n i}$ are lognormal random variables representing the rate of corrosion, and the initiation time of corrosion, respectively. Traffic load distributions are evaluated considering the average daily truck traffic on the bridge based on the load model proposed by Nowak [32]. Analytical and numerical aspects of corrosion and load models are discussed in details in [25, 33]. Random variables involved in the determination of the deck resistance are considered statistically independent from the variables associated with the girders. A coefficient of correlation of 0.7 is considered for the random variables involved in the limit state functions of the four girders. Figure 18(a) shows the annual reliability index for the deck, the interior and the exterior girders of the bridge.

For the evaluation of components and system annual risks, direct consequences have been considered equivalent to rebuilding costs of deck and each girder, assumed as $\$ 100,000$ 
and $\$ 40,000$, respectively. Indirect consequences have been evaluated as the sum of three different contributes: running cost, time loss cost, and safety and environment loss. The first contribute corresponds to the cost of running vehicles along the detour that has to be followed after the bridge failure [34]:

$$
C_{r}=C_{v} L_{d} A_{D T} d_{d}
$$

where $C_{v}$ is the average cost for running a vehicle $(\$ 0.16 / \mathrm{km}), L_{d}$ is the length of the detour (herein considered as $10 \mathrm{~km}$ ), $A_{D T}$ is the average daily traffic (assumed as 400 vehicles per day) and $d_{d}$ is the time period, in days, in which the bridge is not accessible, that, in this case, has been considered as one year.

The time loss cost is the monetary value of the time spent by passengers and trucks to drive through the detour, and it has been estimated based on [34]:

$$
C_{t}=\left[C_{a d} O_{c a r}\left(1-\frac{T_{p}}{100}\right)+C_{t r k} \frac{T_{p}}{100}\right] \frac{L_{d} A_{D T} d_{d}}{s}
$$

where $C_{a d}$ and $C_{t r k}$ are the estimated time values for an adult person (\$7.05 per hour) and trucks (\$20.56 per hour), respectively; $O_{c a r}$ is the average vehicle occupancy in cars (1.56 adult per vehicle); $T_{p}$ is the percentage of trucks on the total number of vehicles (herein assumed as $4 \%$ ); and $s$ is the average speed of the detour $(64 \mathrm{~km} / \mathrm{h})$. Finally, safety and environmental loss should be estimated by a specific analysis that lies outside the scope of this paper; thus, an arbitrary value of $\$ 5 \mathrm{M}$ has been assumed. Direct, indirect and total annual risks for deck, girders and superstructure have been evaluated by means of Eqs. (5) and (6). Total annual risk for the bridge superstructure and its components is shown in Figure 18(b).

Maintenance plans have been evaluated for the described structure considering both a reliability threshold ( $\beta_{t}=3.5$ ) and the associated risk threshold ( $\left.R_{t}=\$ 1,340\right)$, such that they both correspond to the same system failure probability, $P_{f, \text { sys }}=2.3 \cdot 10^{-4}$. Results are shown in Figure 19 in terms of annual reliability and annual risk. Considering the reliability-based approach, exterior girders and deck are repaired simultaneously, because of their lower 
annual reliability index. However, this does not happen in the risk case, since higher priority is given to the deck that is the series component in the system. Over the 70 years of considered life-cycle herein considered, the reliability-based approach returns an additional repair for the exterior girders when compared with the risk-based approach, with only a marginal improvement of the system annual reliability.

Finally, availability and hazard approaches are considered. Deck and girders have been assumed to follow the Weibull distribution (Eq. (23)) considering $\lambda=8 \cdot 10^{-3}, k=2.4$ for the deck; $\lambda=8 \cdot 10^{-3}, k=2.3$ for the exterior girders; and $\lambda=6 \cdot 10^{-3}, k=2.1$ for the interior girders. Figures 20(a) and (b) illustrate availability and hazard functions, respectively, for components and system, considering the two extreme cases of statistically independent and perfectly correlated failure modes, constituting upper and lower bounds for the possible lifetime distributions for the system. Availability and hazard thresholds have been given values $A_{t}=0.95$ and $h_{t}=3 \cdot 10^{-3}$ and corresponding results are shown in Figure 21 and 22 for both statistically independent and perfectly correlated cases. Comparing the two approaches in terms of availability, the hazard approach results to be more conservative for statistically independent failure modes (the minimum value for the corresponding availability is 0.96 , that is higher than the threshold $A_{t}$ ). However, in both cases, only the deck is repaired twice. Conversely, the hazard approach is less conservative for perfectly correlated failure modes, when the availability reaches the value of 0.94 , although it requires one additional repair for the deck.

\section{Conclusions}

Two annual performance indicators, namely annual reliability index and annual risk, and two lifetime distributions (availability and hazard functions) have been considered to define system threshold-based approaches for the determination of maintenance times of deteriorating structural systems. Different system models of the same components have been analyzed, as well as different correlation among structural failure modes. The approach presented has broad applicability to any structure that can be adequately modeled as a system 
of interrelated components such as highway bridges, naval vessels, and aerospace structures. The conclusions are as follows:

1. Since annual reliability index and annual risk are both strictly connected with the system failure probability, similar trends have to be expected in the timing for maintenance produced by these two approaches. In particular, when analyzing systems with perfectly correlated failure modes, reliability and riskbased approaches provide the same results for all system models considered.

2. Differences in results provided by risk and reliability-based methods are observed when failure modes are not perfectly correlated and consequences associated with single components are significantly different. In these cases, the risk-based approach gives higher maintenance priority to the component with highest associated annual risk, allowing a reduction of the number of required repairs over the service life-cycle of deteriorating structures.

3. Although approaches based on lifetime distributions are somehow limited to the extreme cases of independence or perfect correlation among failure modes, the possibility of defining importance factors allows to choose a priori which component has to be repaired to obtain the maximum effect on the system reliability. Therefore, maintenance schedules obtained through this kind of approach require, in general, a lower number of repairs compared to the reliability-based approach.

4. There is not a direct relation between availability and hazard thresholds, since the two approaches aim at controlling different aspects of the problem (probability of being functional and rate of failure, respectively). However, the hazard-based method seems to be more conservative than the availability method, when no correlation among failure modes is present.

Certainly, further research is needed on decision making based on combining several indicators at the same time (reliability and risk, availability and hazard) through multiobjective optimization methods, involving also minimization of maintenance cost. 


\section{Acknowledgments}

The support by grants from (a) the National Science Foundation (NSF) Award CMS-0639428, (b) the Commonwealth of Pennsylvania, Department of Community and Economic Development, through the Pennsylvania Infrastructure Technology Alliance (PITA), (c) the U.S. Federal Highway Administration (FHWA) Cooperative Agreement Award DTFH61-07H-00040, (d) the U.S. Office of Naval Research (ONR) Awards N00014-08-1-0188 and N00014-12-1-0023 and (e) the National Aeronautics and Space Administration (NASA) Award NNX10AJ20G is gratefully acknowledged. The opinions presented in this paper are those of the authors and do not necessarily reflect the views of the sponsoring organizations.

\section{References}

[1] Ellingwood, B.R. (2005), "Risk-informed condition assessment of civil infrastructure: state of practice and research issues”, Structure and Infrastructure Engineering, 1(1), 718.

[2] Frangopol, D.M. (2011), "Life-cycle performance, management and optimisation of structural systems under uncertainty: accomplishments and challenges”, Structure and Infrastructure Engineering, 7(6), 389-413.

[3] CIB Working Commission TG32 (Public Perception of Safety and Risks in Civil Engineering) (2001), "Risk assessment and risk communication in civil engineering” CIB Report, Publication 259.

[4] Maes, M.A., Faber, M.H. (2008), "Utility, preferences, and risk perception in engineering decision making”, JCSS(Join Committee of Structural Safety) Document No. 6, November 2008. 
[5] Faber, M.H. (2003), “Uncertainty modeling and probabilities in engineering decision analysis”, Proceeding of the International Conference on Offshore Mechanics and Arctic Engineering, OMAE2003, Cancun, Mexico.

[6] Joint Committee on Structural Safety (2008), "Risk assessment in engineering principles, system representation \& risk criteria”, ISBN 978-3-909386-78-9.

[7] Saydam, D., Frangopol, D.M. (2011), “Time-dependent performance indicators of damaged bridge superstructures”, Engineering Structures, 33, 2458-2471.

[8] Estes, A.C., Frangopol, D.M. (1999), "Repair optimization of highway bridges using system reliability approach”, Journal of Structural Engineering, 125(7), 766-775.

[9] Kim, S., Frangopol, D.M., Zhu, B. (2011), "Probabilistic optimum inspection/repair planning to extend lifetime of deteriorating structures”, Journal of Performance of Constructed Facilities, 25, 535-544.

[10] Onoufriou, T. (1999), “Reliability based inspection planning of offshore structures”, Marine Structures, 12, 521-539.

[11] Moan, T. (2005), "Reliability-based management of inspection, maintenance and repair of offshore structures”, Structure and Infrastructure Engineering, 1(1), 33-62.

[12] Mori, Y., Ellingwood, B.R. (1994), “Maintaining reliability of concrete structures. II: optimum inspection/repair”, Journal of Structural Engineering, 120(3), 846-862.

[13] Orcesi, A.D., Frangopol, D.M. (2011), “A stakeholder probability-based optimization approach for cost-effective bridge management under financial constraints”, Engineering Structures, 33, 1439-1449. 
[14] Ang, A.H-S., Tang, W.H. (1984), "Probability concepts in engineering planning and design. Volume II - Decision, risk and reliability”, J. Wiley and Sons, NY, USA.

[15] Yang, S., Frangopol, D.M., Neves, L.C. (2006), “Optimum maintenance strategy for deteriorating bridge structures based on lifetime functions”, Engineering Structures, 28, 196-206.

[16] Okasha, N.M., Frangopol, D.M. (2010a), "Novel approach for multicriteria optimization of life-cycle preventive and essential maintenance of deteriorating structures”, Journal of Structural Engineering, 138(8), 1009-1022.

[17] Arunraj, N.S., Maiti, J. (2007), "Risk-based maintenance - Techniques and applications”, Journal of Hazardous Materials, 142, 653-661.

[18] Hessami, A.G. (1999), "Risk management: a systems paradigm”, Systems Engineering, 2(3), 156-167.

[19] Okasha, N.M., Frangopol, D.M. (2010c), “Time-variant redundancy of structural systems”, Structure and Infrastructure Engineering, 6(1-2), 279-301.

[20] Estes, A.C., Frangopol, D.M. (1998), "RELSYS: A computer program for structural system reliability analysis”, Structural Engineering Mechanics, 6(8), 901-919.

[21] Okasha, N.M., Frangopol, D.M. (2010b), "Redundancy of structural systems with and without maintenance: an approach based on lifetime functions”, Reliability Engineering and System Safety, 95, 520-533. 
[22] Leemis, L.M. (1995), "Reliability, probabilistic models and statistical methods”, NJ, USA, Prentice Hall.

[23] Barone, G., Frangopol, D.M. (2013), “Hazard-based optimum lifetime inspection/repair planning for deteriorating structures”, Journal of Structural Engineering, doi:10.1061/(ASCE)ST.1943-541X.0000810.

[24] Barone, G., Frangopol, D.M., Soliman, M. (2013), "Optimization of life-cycle maintenance of deteriorating bridges considering expected annual system failure rate and expected cumulative cost”, Journal of Structural Engineering, doi:10.1061/(ASCE)ST.1943-541X.0000812.

[25] Akgül, F. (2002), "Lifetime system reliability prediction for multiple structure types in a bridge network”, PhD Thesis, Department of Civil, Environmental, and Architectural Engineering, University of Colorado, Boulder, Colorado.

[26] Marsh, P.S., Frangopol, D.M., (2008), "Reinforced concrete bridge deck reliability model incorporating temporal and spatial variations of probabilistic corrosion rate sensor data”, Reliability Engineering and System Safety, 93, 394-409.

[27] Bertolini, L., (2008), "Steel corrosion and service life of reinforced concrete structures”, Structure and Infrastructure Engineering, 4(2), 123-137.

[28] Collepardi, M., Marcialis, A., Turriziani, R. (1972), "Penetration of chloride ions into cement pastes and concretes”, Journal of The American Ceramic Society, 55(10), 534535. 
[29] Garbatov, Y., Guedes Soares , C., Wang, G. (2007), “Nonlinear time dependent corrosion wastage of deck plates of ballast and cargo tanks of tankers”, Journal of Offshore Mechanics and Arctic Engineering, 129, 48-55.

[30] Guedes Soares, C., Garbatov, Y., Zayed A., Wang, G. (2009), "Influence of environmental factors on corrosion of ship structures in marine atmosphere”, Corrosion Science, 51, 2014-2026.

[31] Garbatov, Y., Guedes Soares, C. (2009), "Structural maintenance planning based on historical data of corroded deck plates of tankers”, Reliability Engineering and System Safety, 94(11), 1806-1877.

[32] Nowak, A.S., (1993), "Live load model for highway bridges”, Structural Safety, 13, 5366.

[33] Estes, A.C. (1997), “A system reliability approach to the lifetime optimization of inspection and repair of highway bridges”, PhD Thesis, Department of Civil, Environmental, and Architectural Engineering, University of Colorado, Boulder, Colorado.

[34] Stein, S.M., Young, G.K., Trent, R.E., Pearson, D.R. (1999), "Prioritizing scour vulnerable bridges using risk”, Journal of Infrastructure Systems, 5(3), 95-101. 


\section{Table 1}

Mean $\mu$ and standard deviation $\sigma$ of the random variables associated with the definition of the bridge performance functions (based on Akgül 2002 [25]).

\begin{tabular}{llll|llll}
\hline Variables & Dimensions & $\mu$ & $\sigma$ & Variables & Dimensions & $\mu$ & $\sigma$ \\
\hline$f_{y, d}$ & $M P a$ & 309 & 34 & $f_{y, g}$ & $M P a$ & 309 & 34 \\
$f_{c, d}$ & $M P a$ & 19 & 3.4 & $f_{c, g}$ & $M P a$ & 19 & 3.4 \\
$\lambda_{d}$ & 1 & 0.02 & $\lambda_{d, i}$ & & 1 & 0.02 \\
$\gamma_{d}$ & & 1.02 & 0.06 & $\gamma_{g}$ & & 1.02 & 0.06 \\
$I_{f}$ & 1.15 & 0.12 & $D_{f}$ & & 1.44 & 0.18 \\
$r_{\text {deck }}, r_{g, \text { int }}$ & mm/year & 0.076 & 0.002 & $r_{g, \text { ext }}$ & mm/year & 0.15 & 0.002 \\
$T_{\text {ini }}$ & years & 9.96 & 4.75 & & & & \\
\hline
\end{tabular}




\section{Figure captions}

Figure 1 Resistance, load effect and maintenance cost over time.

Figure 2 Three-component system configurations.

Figure 3 Annual failure probability and annual reliability index for systems A-D in Figure 2: (a)-(b) statistically independent and (c)-(d) perfectly correlated failure modes.

Figure 4 Annual risk for systems A-D IN Figure 2 considering statistically independent failure modes.

Figure 5 Annual risk for systems A-D in Figure 2 considering perfectly correlated failure modes.

Figure 6 Reliability-based and risk-based maintenance schedules considering statistical independent failure modes. Systems A, C, and D in Figure 2.

Figure 7 Reliability-based and risk-based maintenance schedules considering perfectly correlated failure modes. Systems A-D in Figure 2.

Figure 8 Essential maintenance considering reliability-based threshold $\left(\beta_{t, 1}\right)$. System C with (a)-(b) statistically independent and (c)-(d) perfectly correlated failure modes.

Figure 9 Essential maintenance considering risk threshold $\left(R_{t, 1}\right)$; System C with (a)-(b) statistically independent and (c)-(d) perfectly correlated failure modes. 
Figure 10 Maintenance cost profiles for systems A, C, and D.

Figure 11 Survivor and hazard functions for systems A-C, (a)-(b) statistically independent and (c)-(d) perfectly correlated failure modes.

Figure 12 Availability importance factors considering independent failure modes of systems A, B and C in Figure 2.

Figure 13 Availability-based and hazard-based maintenance schedules considering statistical independent failure modes. Systems A and C.

Figure 14 Availability-based and hazard-based maintenance schedules considering perfectly correlated failure modes. Systems A-C.

Figure 15 Essential maintenance considering availability threshold $\left(A_{t, 1}\right)$ : system $\mathrm{C}$ in Figure 2 with (a)-(b) statistically independent and (c)-(d) perfectly correlated failure modes.

Figure 16 Essential maintenance considering hazard threshold $\left(h_{t, 1}\right)$ : system $C$ in Figure 2 with (a)-(b) statistically independent and (c)-(d) perfectly correlated failure modes.

Figure 17 Bridge superstructure cross-section (adapted from Akgul 2002 [18]) and its series-parallel model.

Figure 18 Bridge superstructure: (a) annual reliability index and (b) risk with no maintenance. 
Figure 19 Bridge superstructure: (a)-(b) reliability-based maintenance and (c)-(d) riskbased maintenance.

Figure 20 Bridge superstructure: (a) availability and (b) hazard functions.

Figure 21 Essential maintenance considering availability threshold $\left(A_{t}\right)$ : bridge superstructure considering (a)-(b) statistically independent and (c)-(d) perfectly correlated failure modes.

Figure 22 Essential maintenance considering hazard threshold $\left(h_{t}\right)$ : bridge superstructure considering (a)-(b) statistically independent and (c)-(d) perfectly correlated failure modes. 


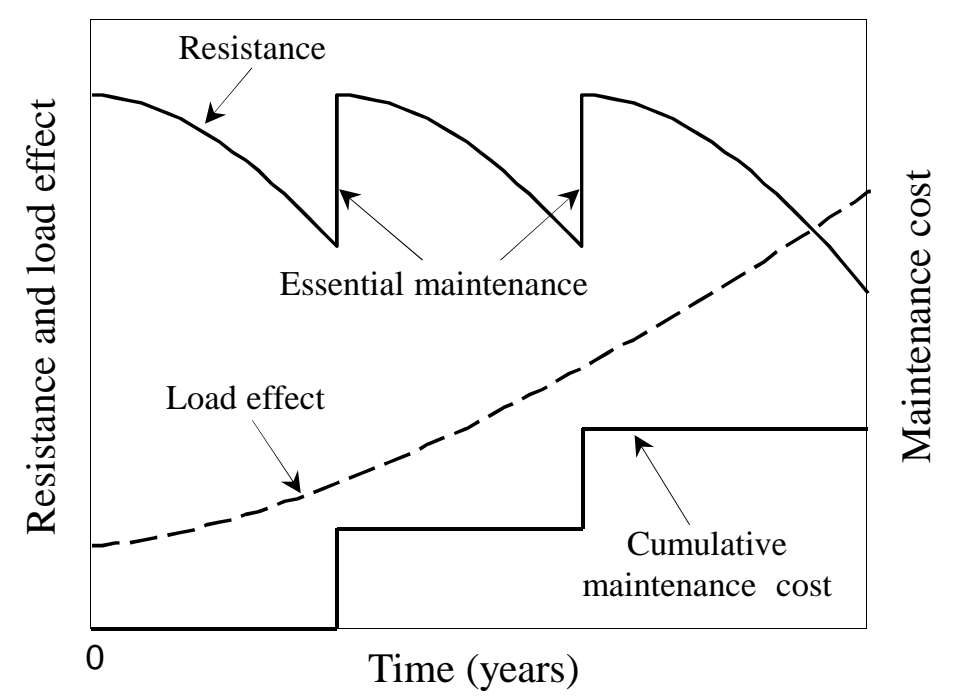


(a)

\section{CONFIGURATION A}

Component 1

Component 2

Component 3

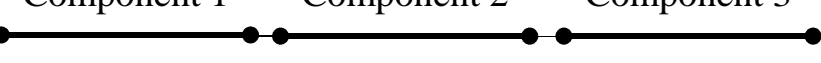

(c)

CONFIGURATION C

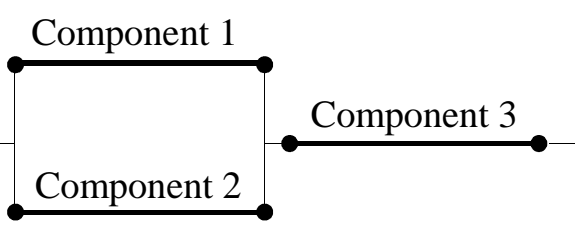

(b)

$$
\text { CONFIGURATION B }
$$

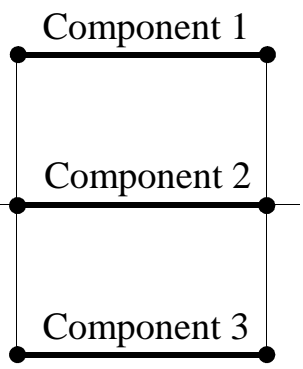

(d)

\section{CONFIGURATION D}

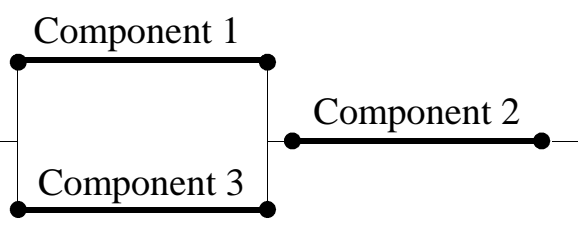


(a)

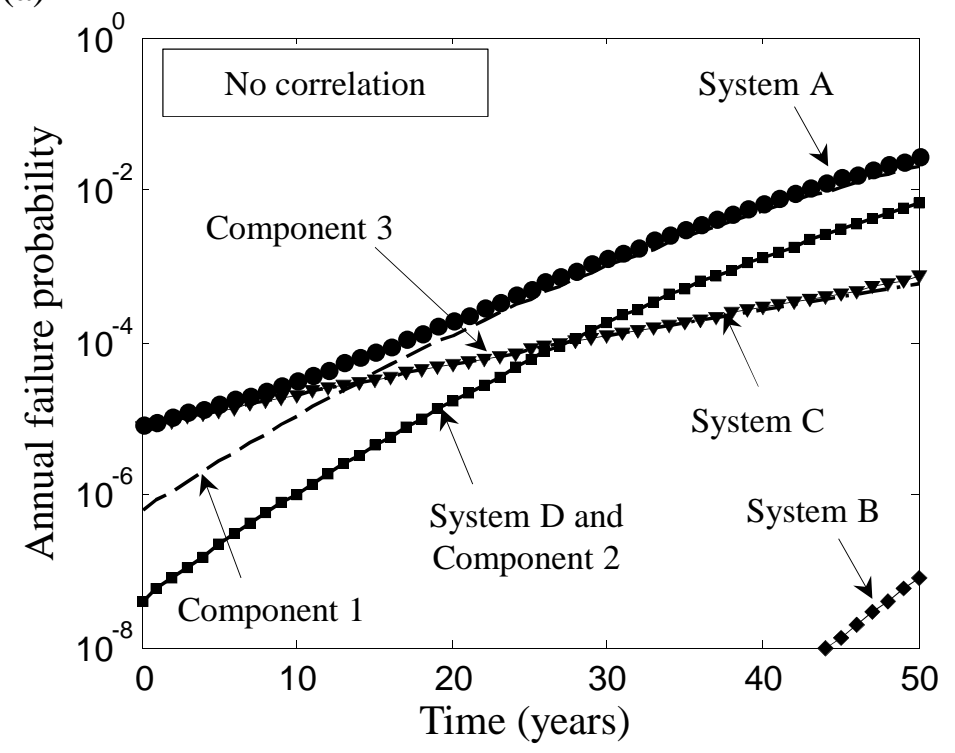

(c)

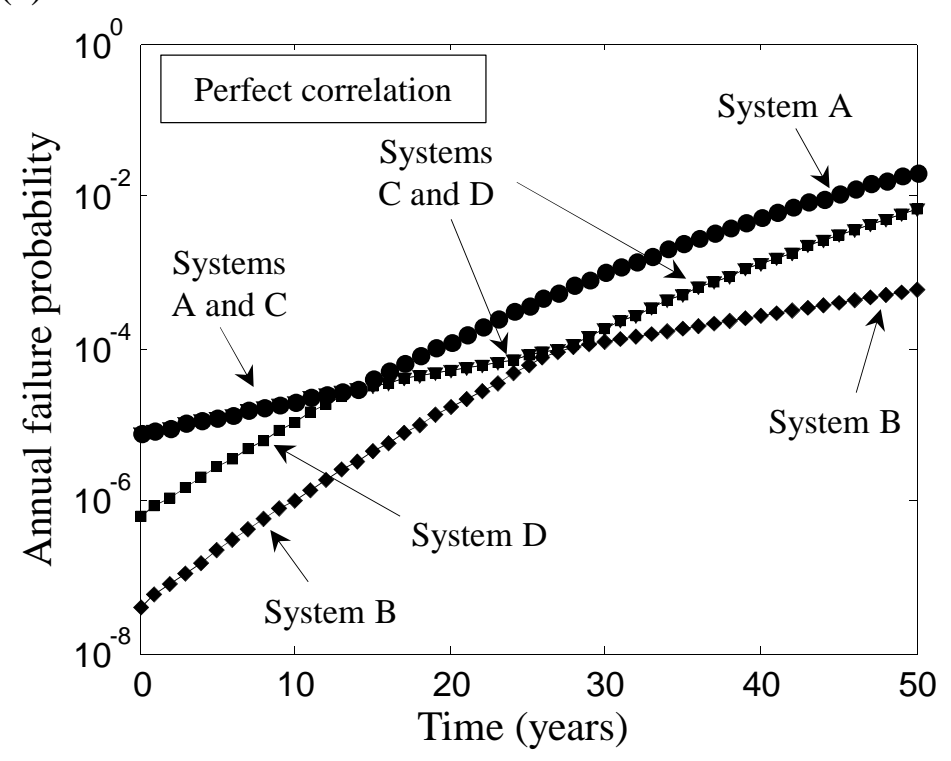

(b)

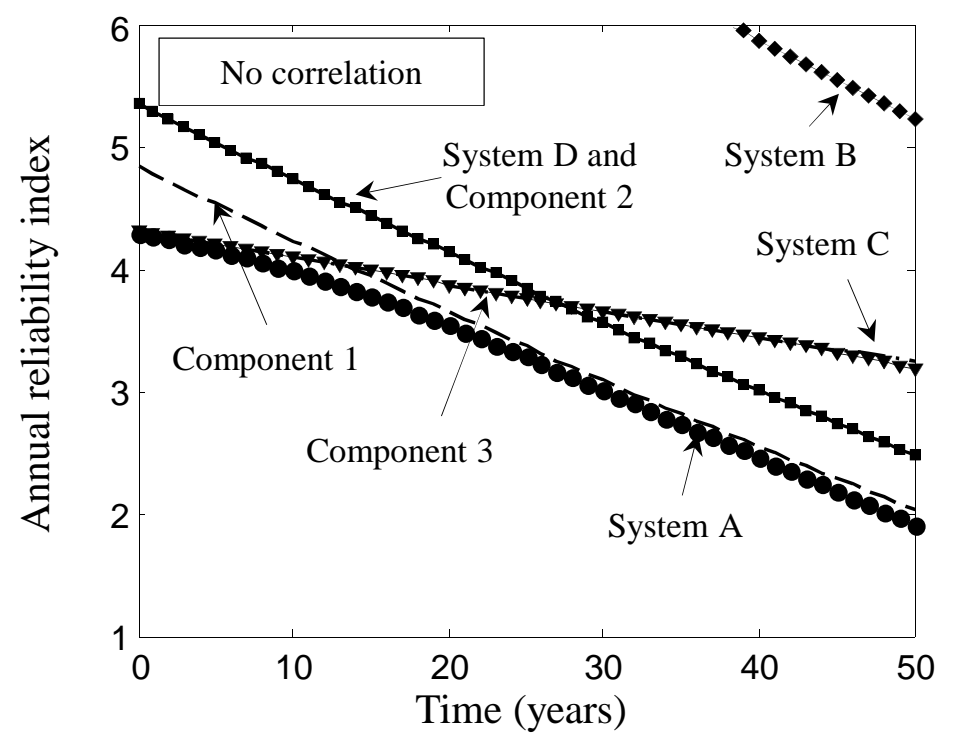

(d)

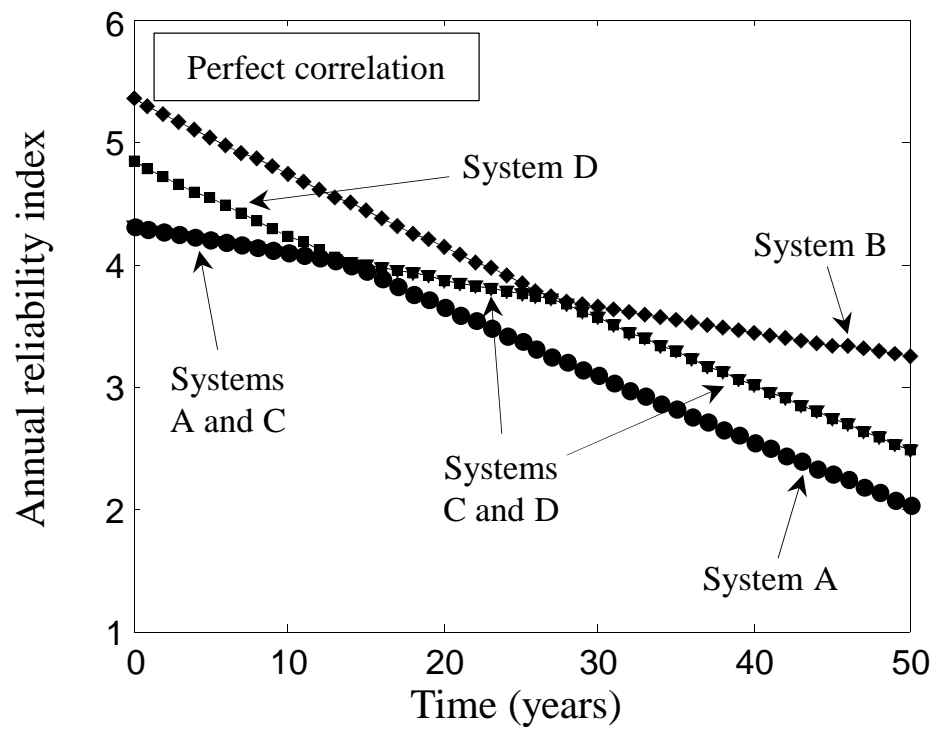


(a)

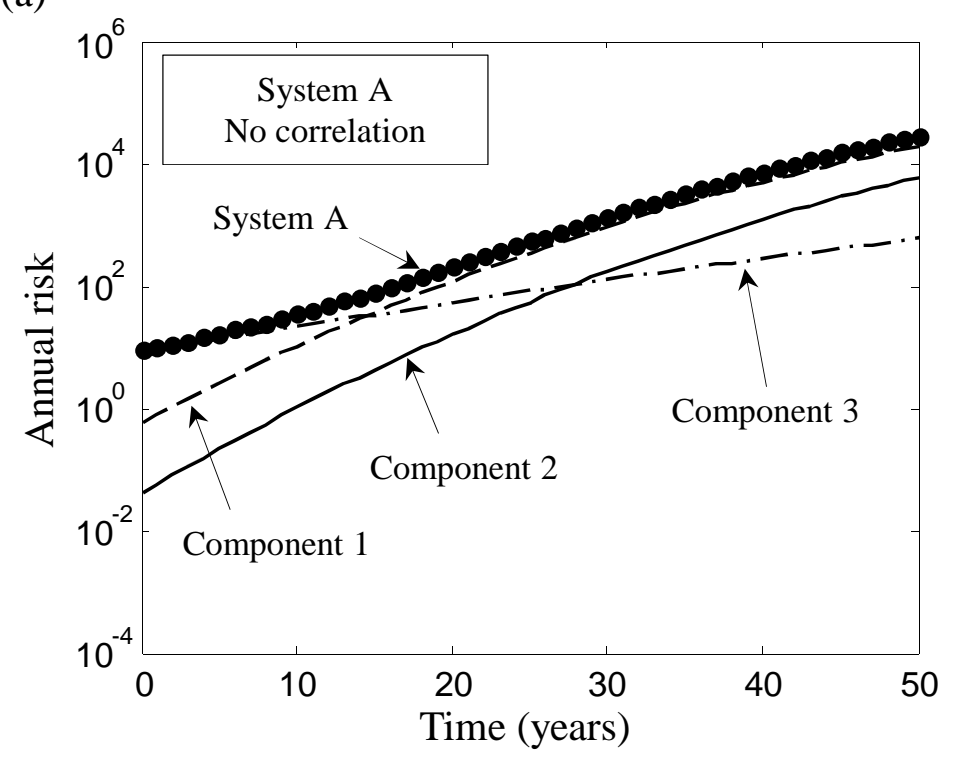

(c)

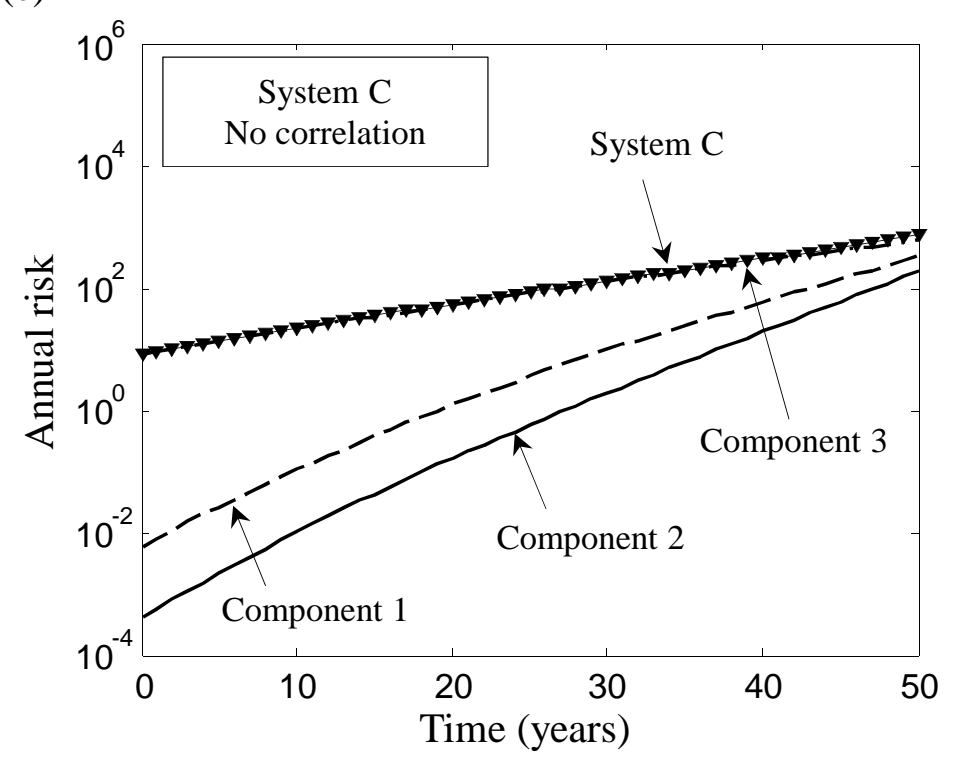

(b)

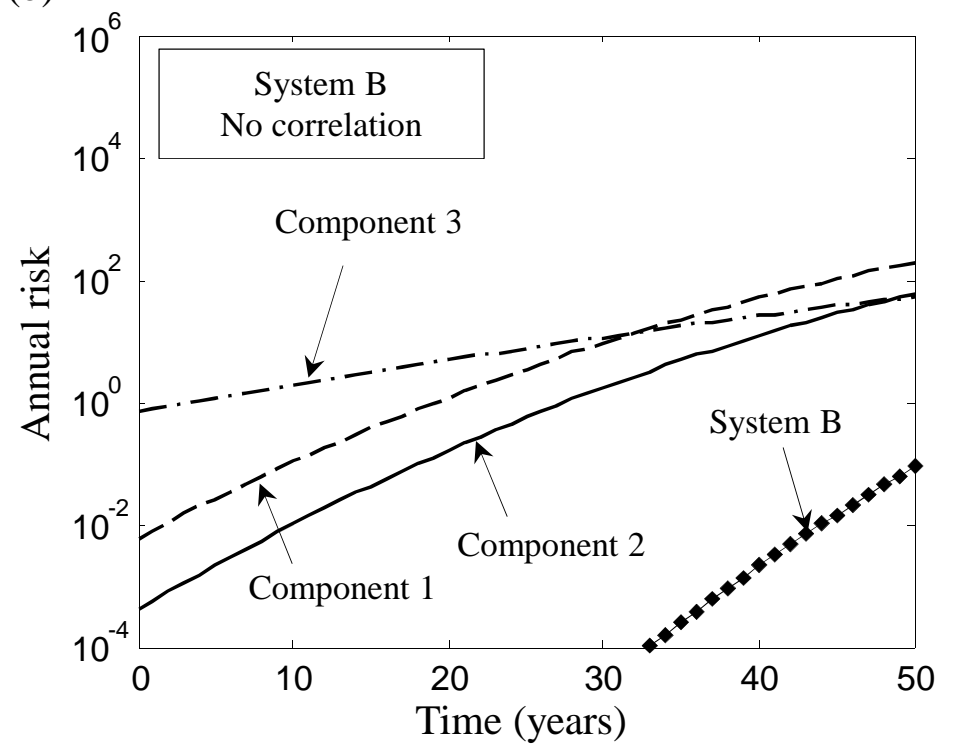

(d)

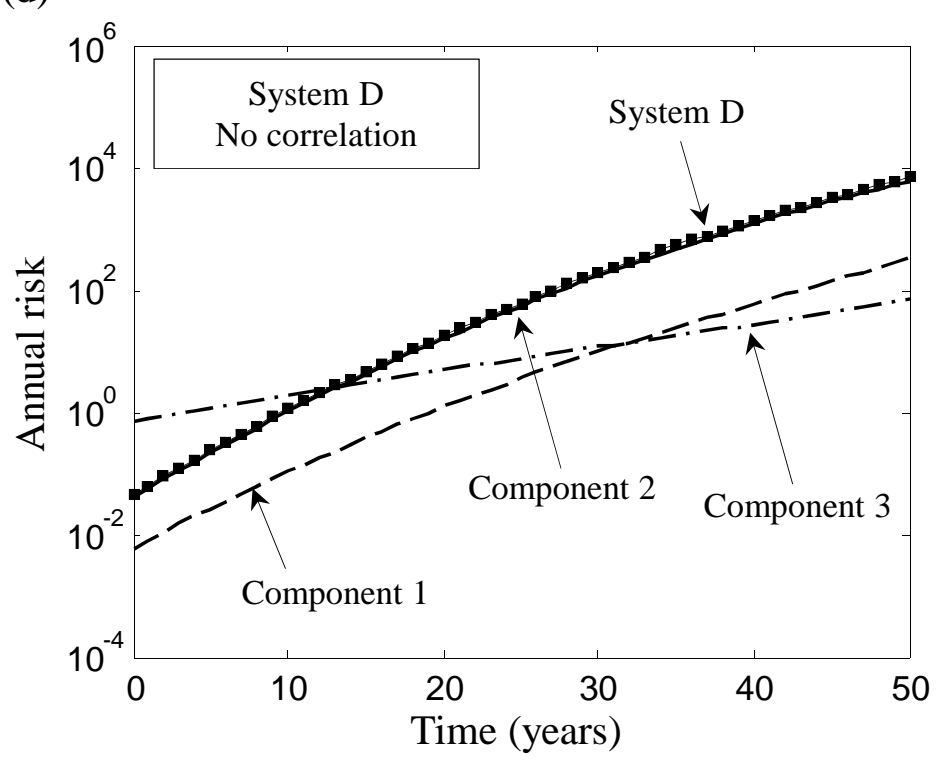


(a)

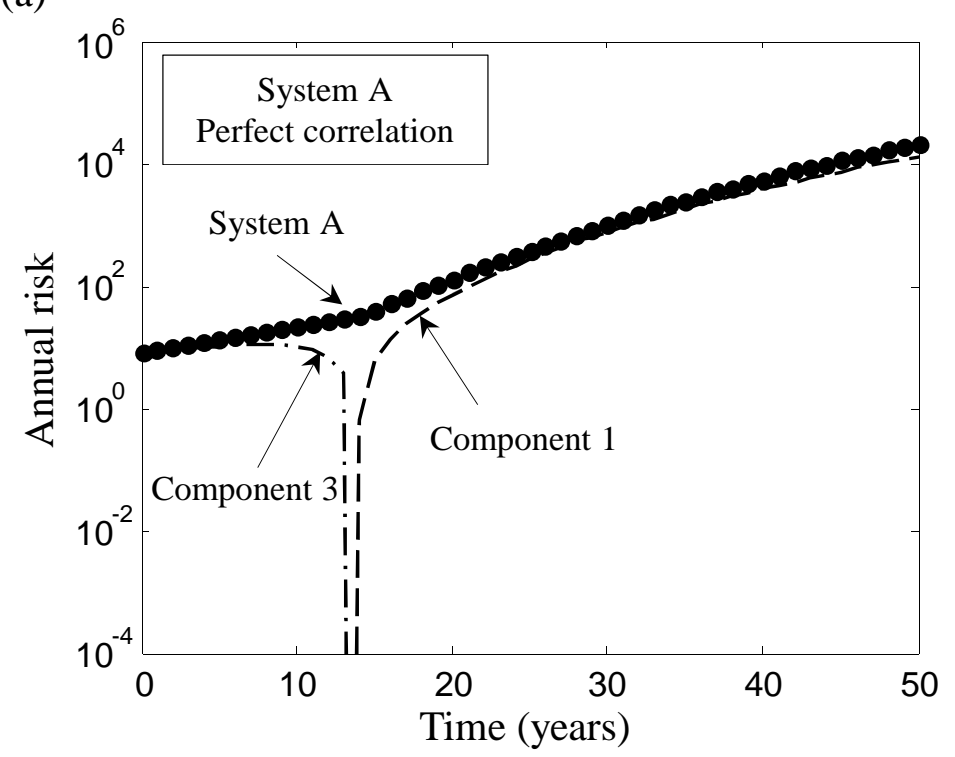

(c)

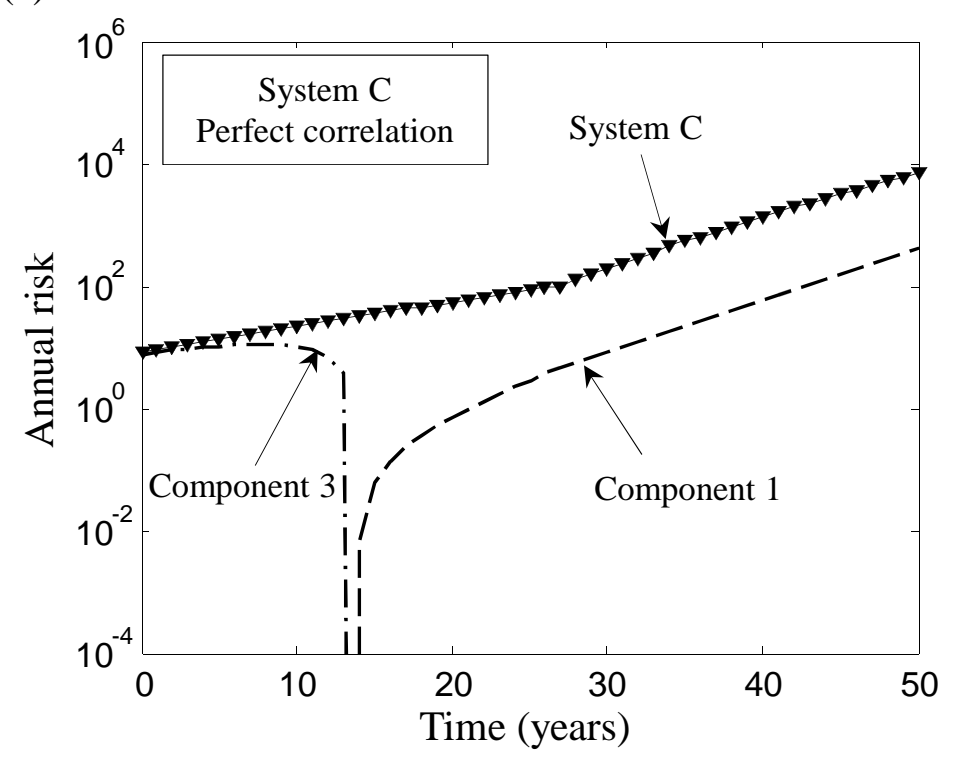

(b)

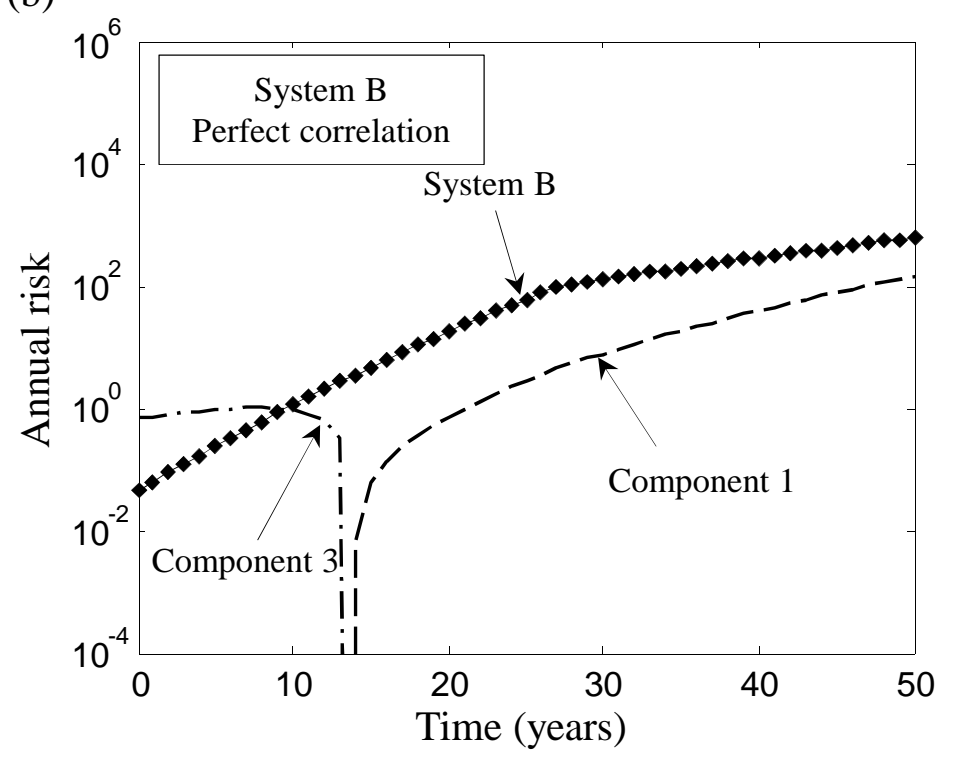

(d)

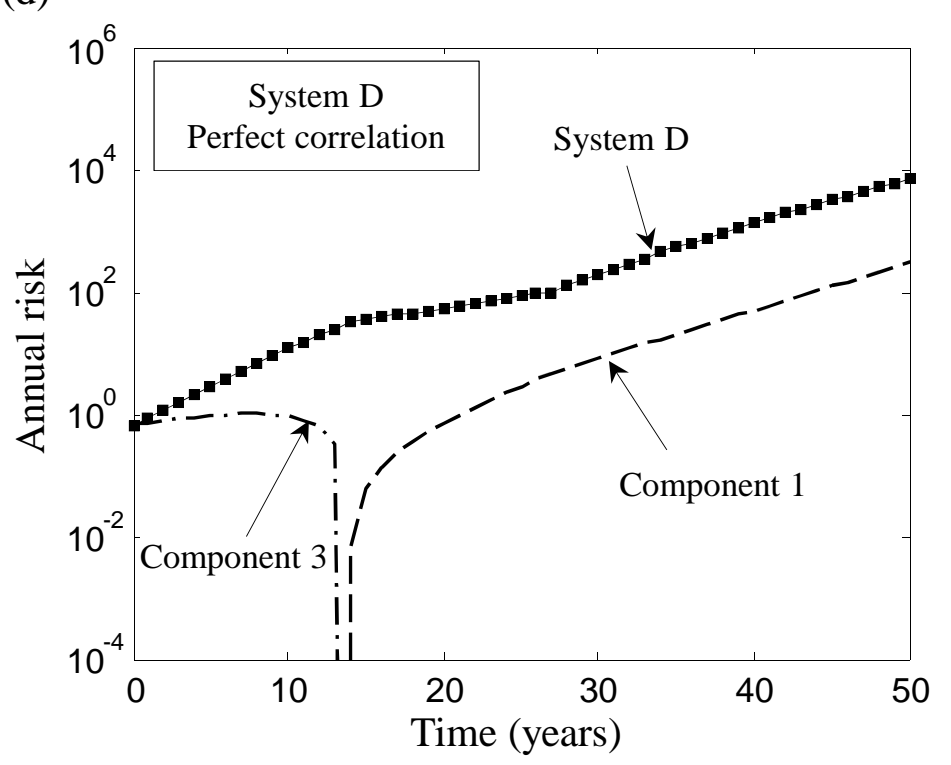




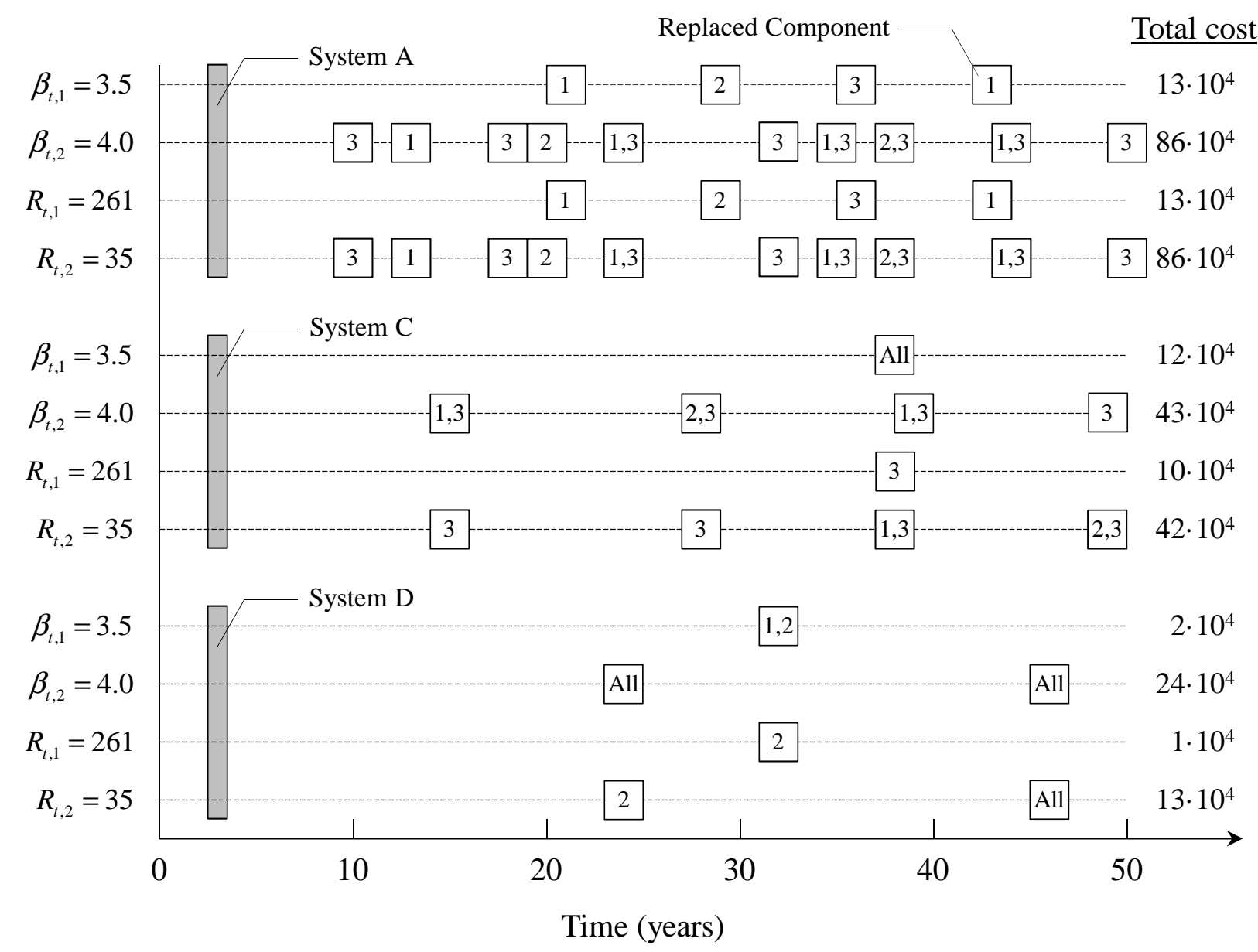




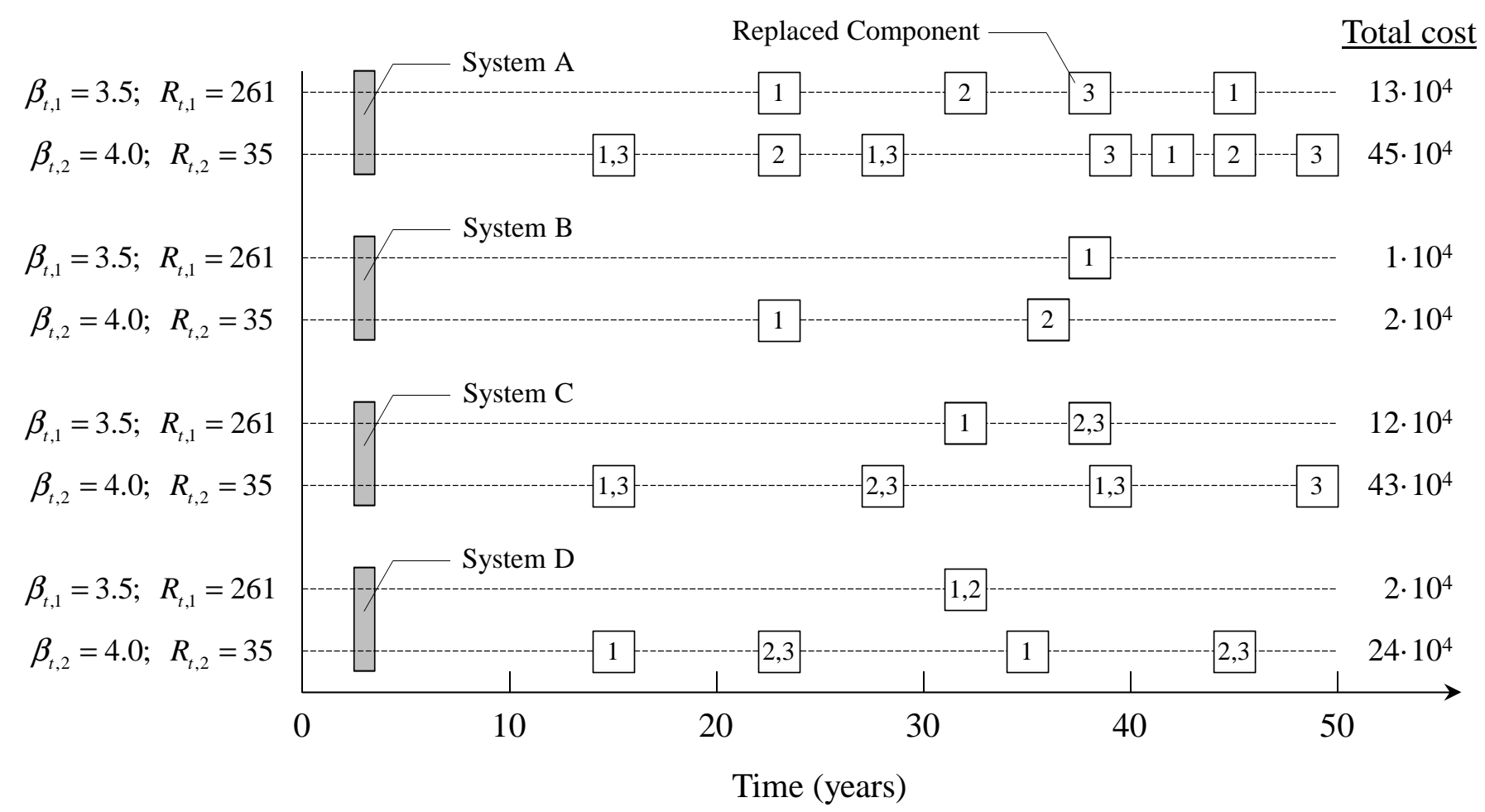


(a)

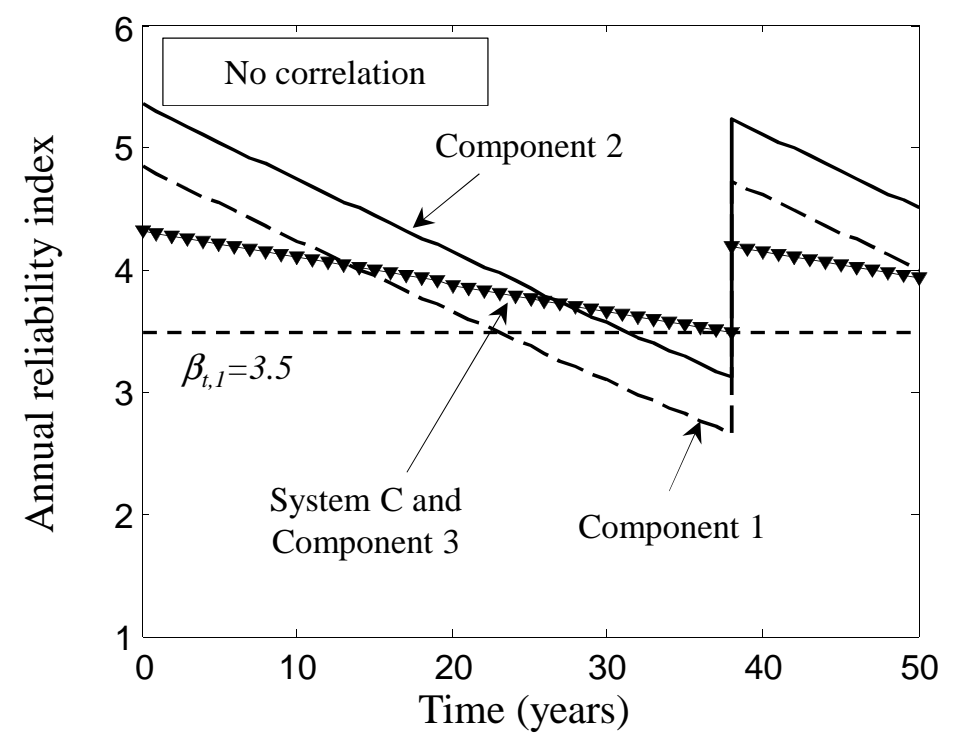

(c)

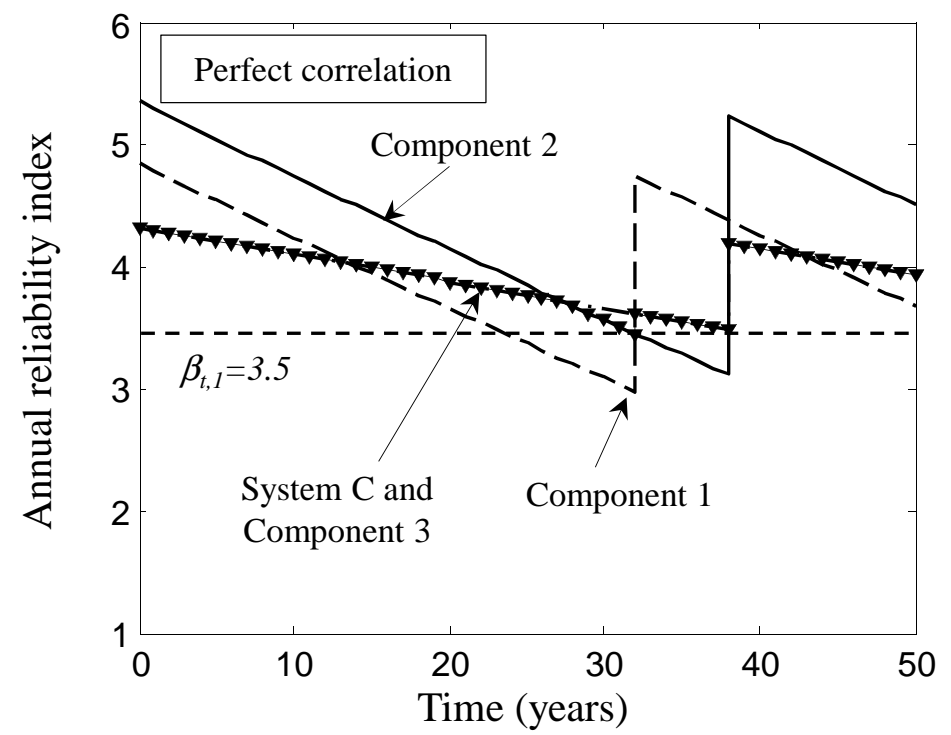

(b)

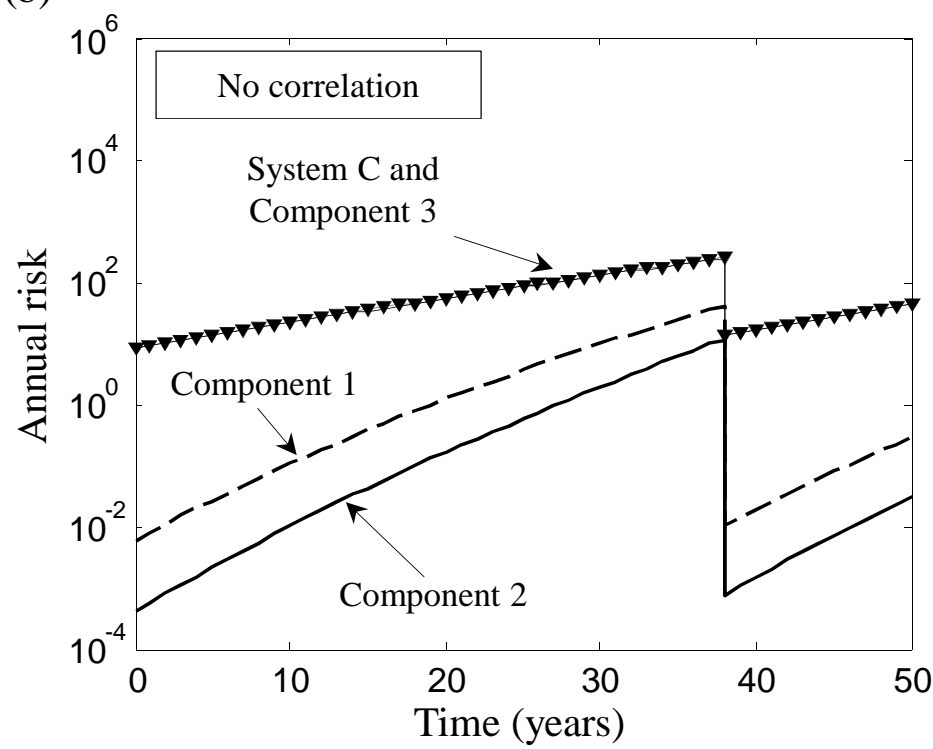

(d)

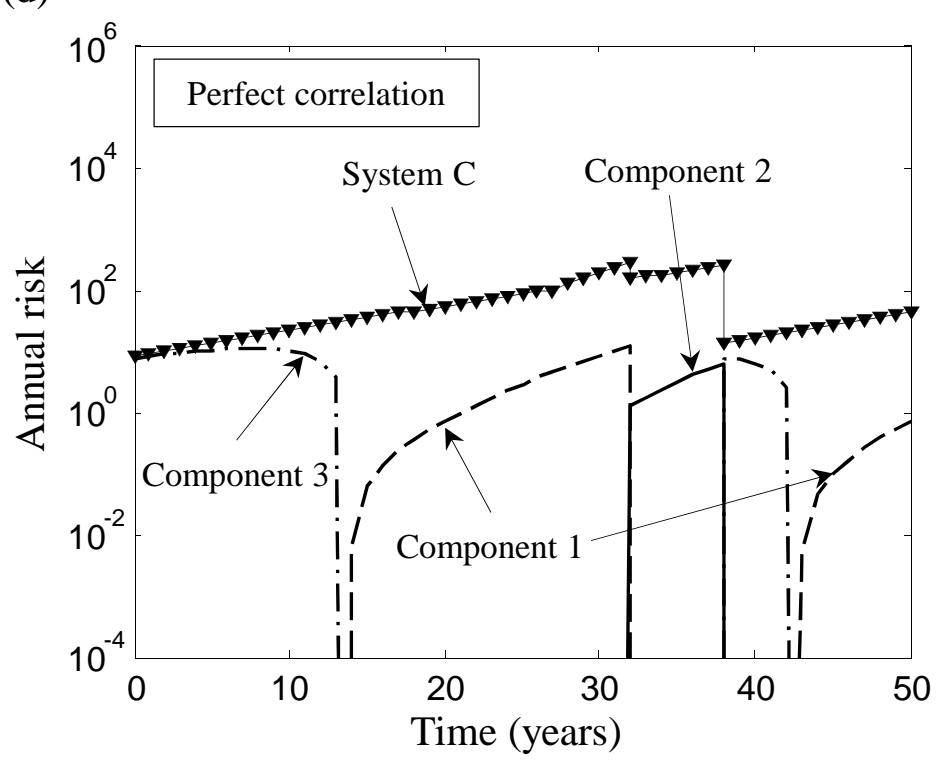


(a)

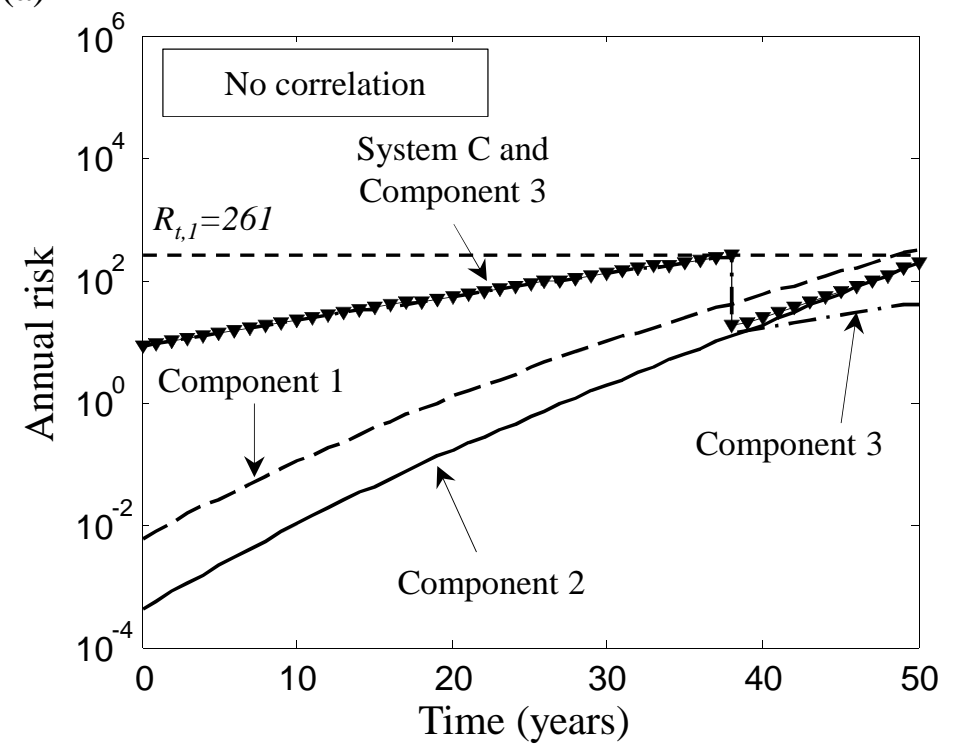

(c)

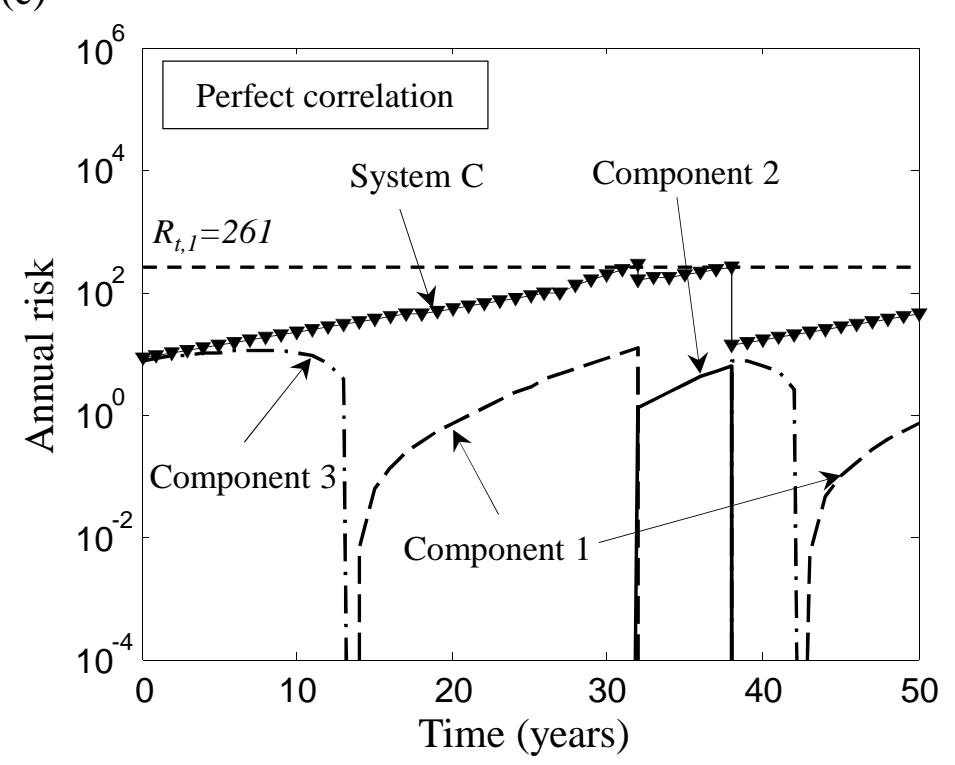

(b)

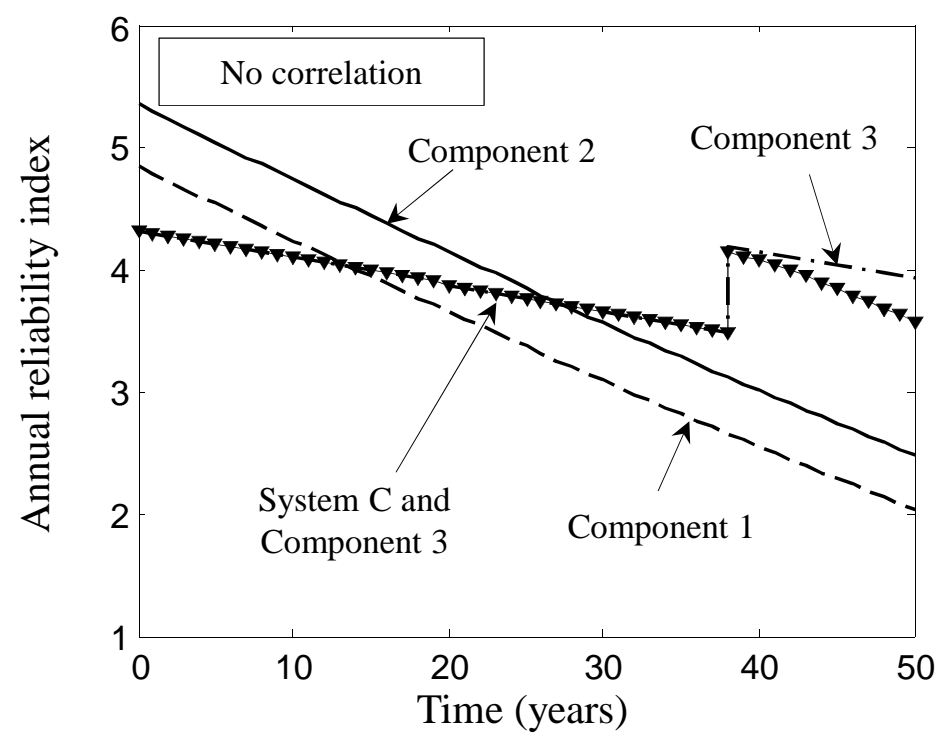

(d)

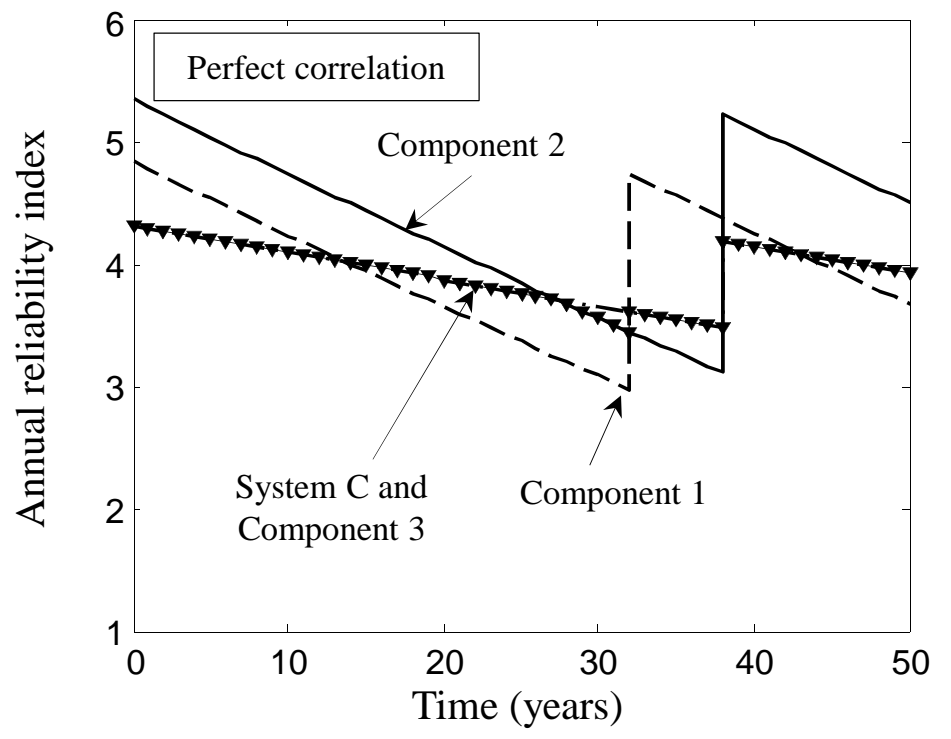



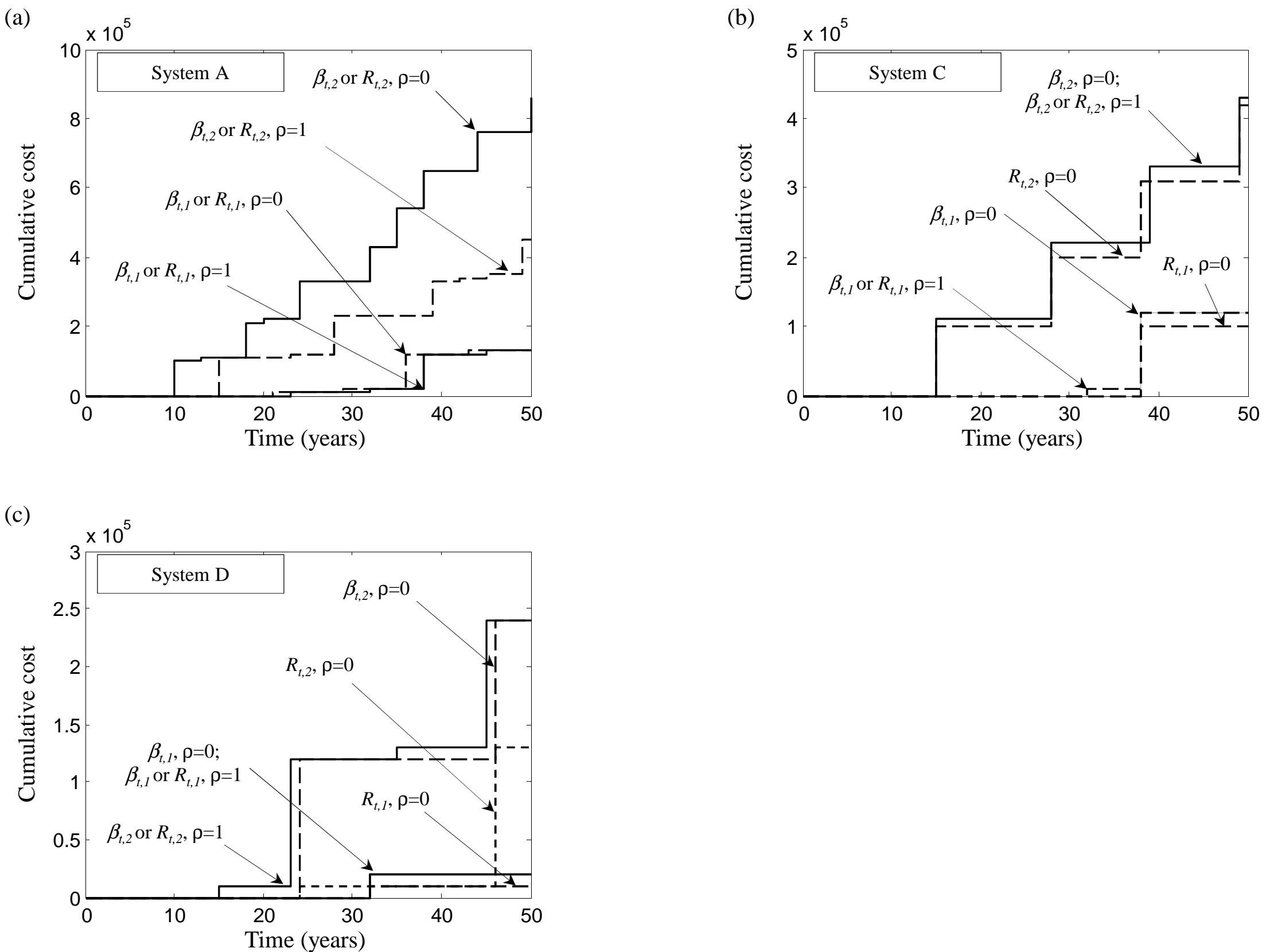
(a)

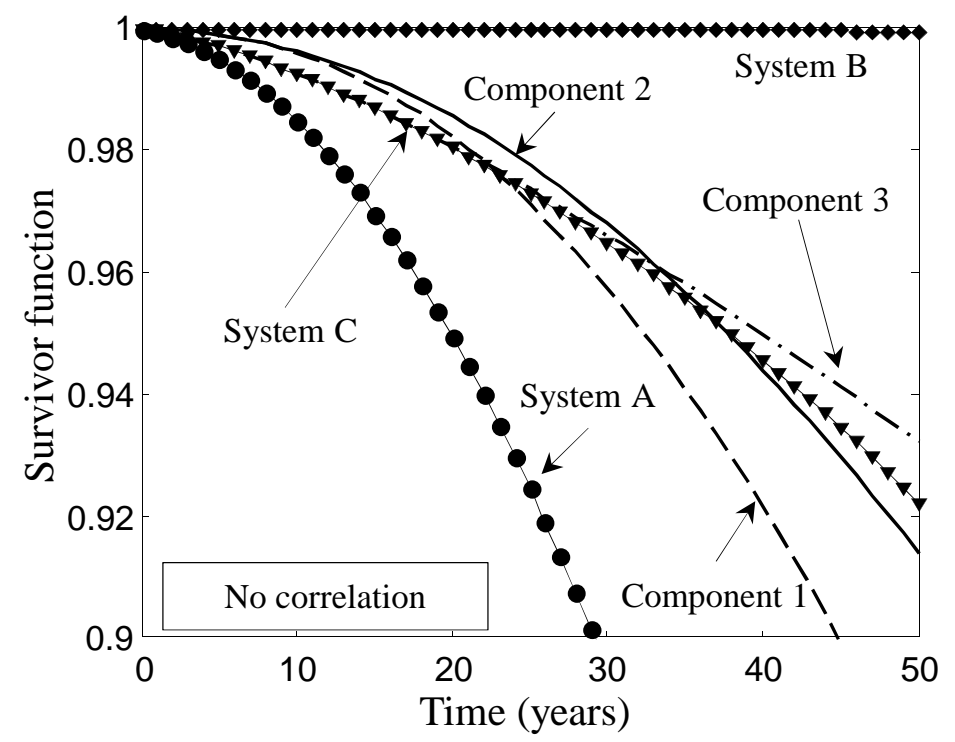

(c)

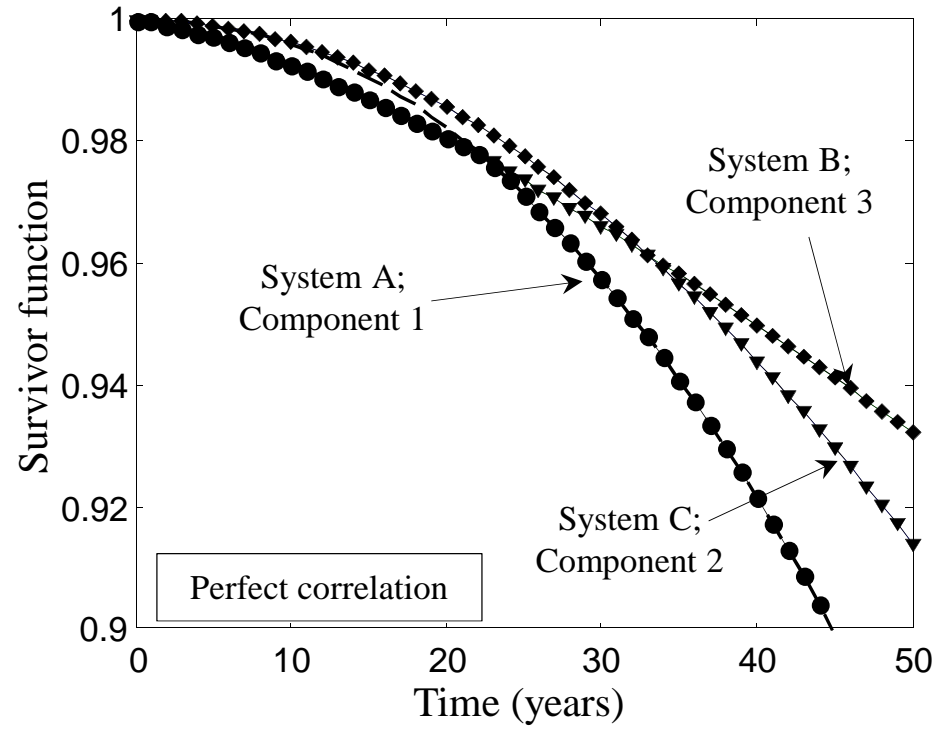

(b)

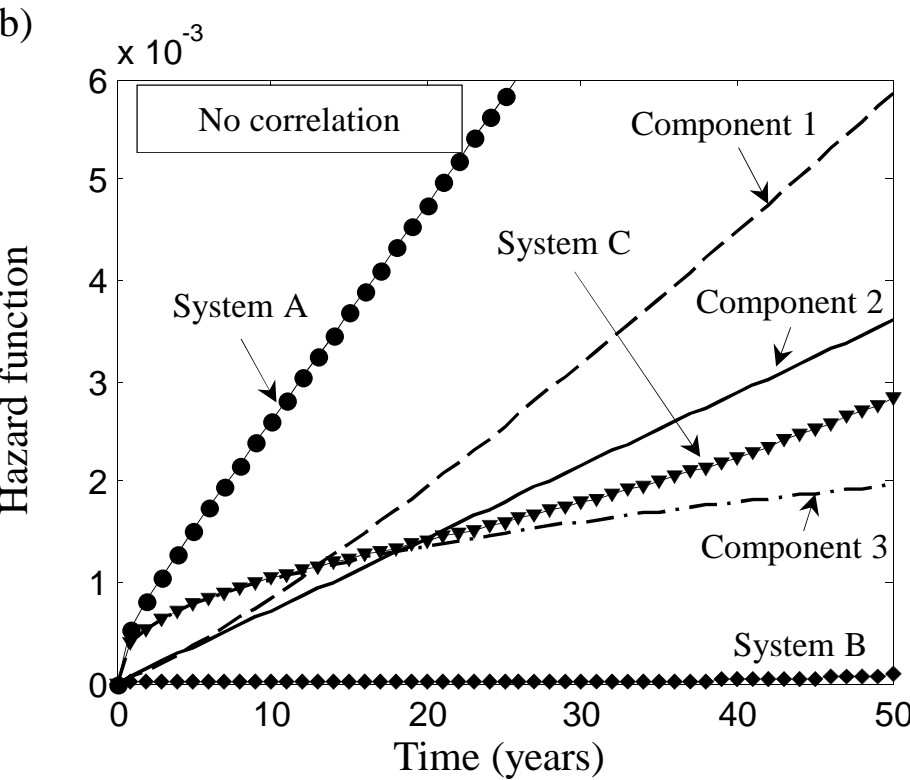

(d)

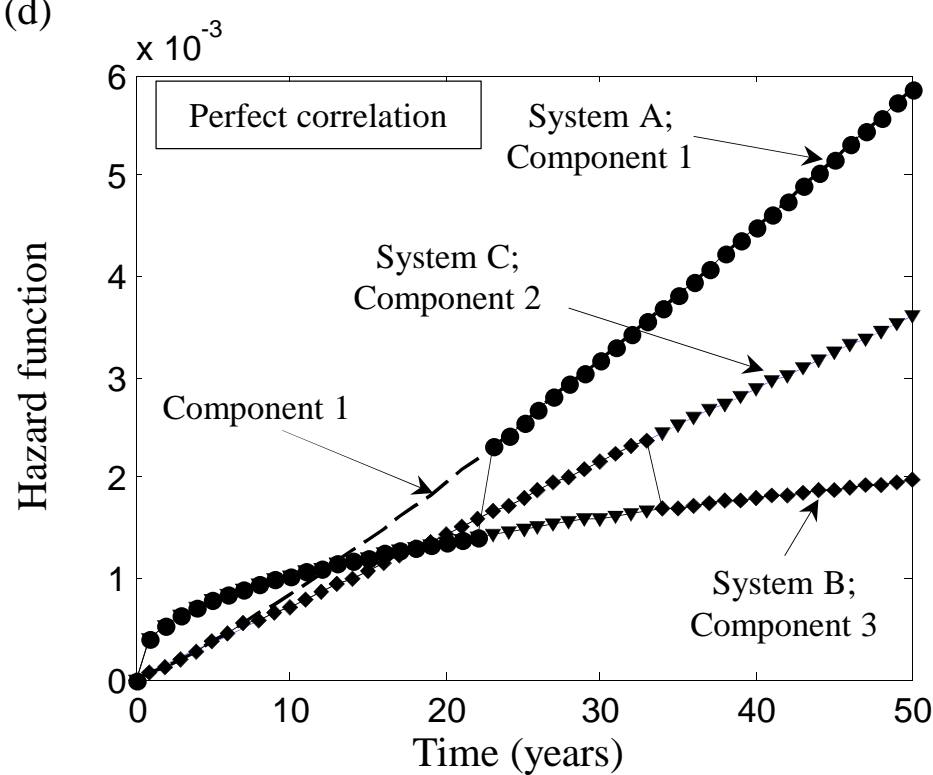


(a)

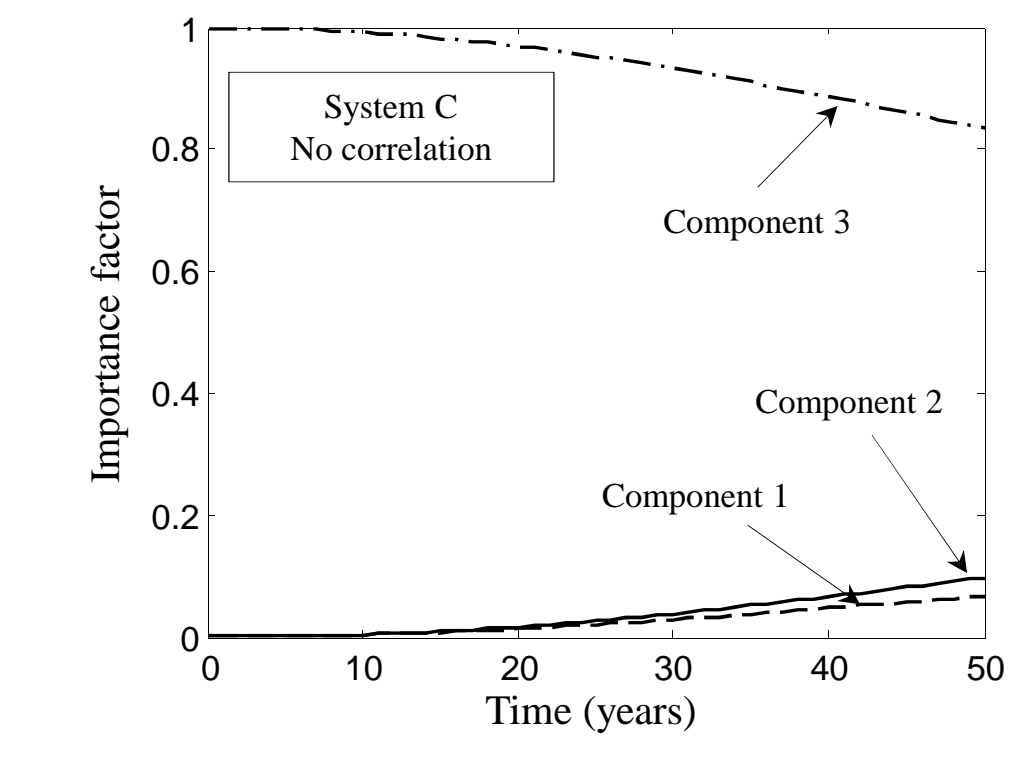

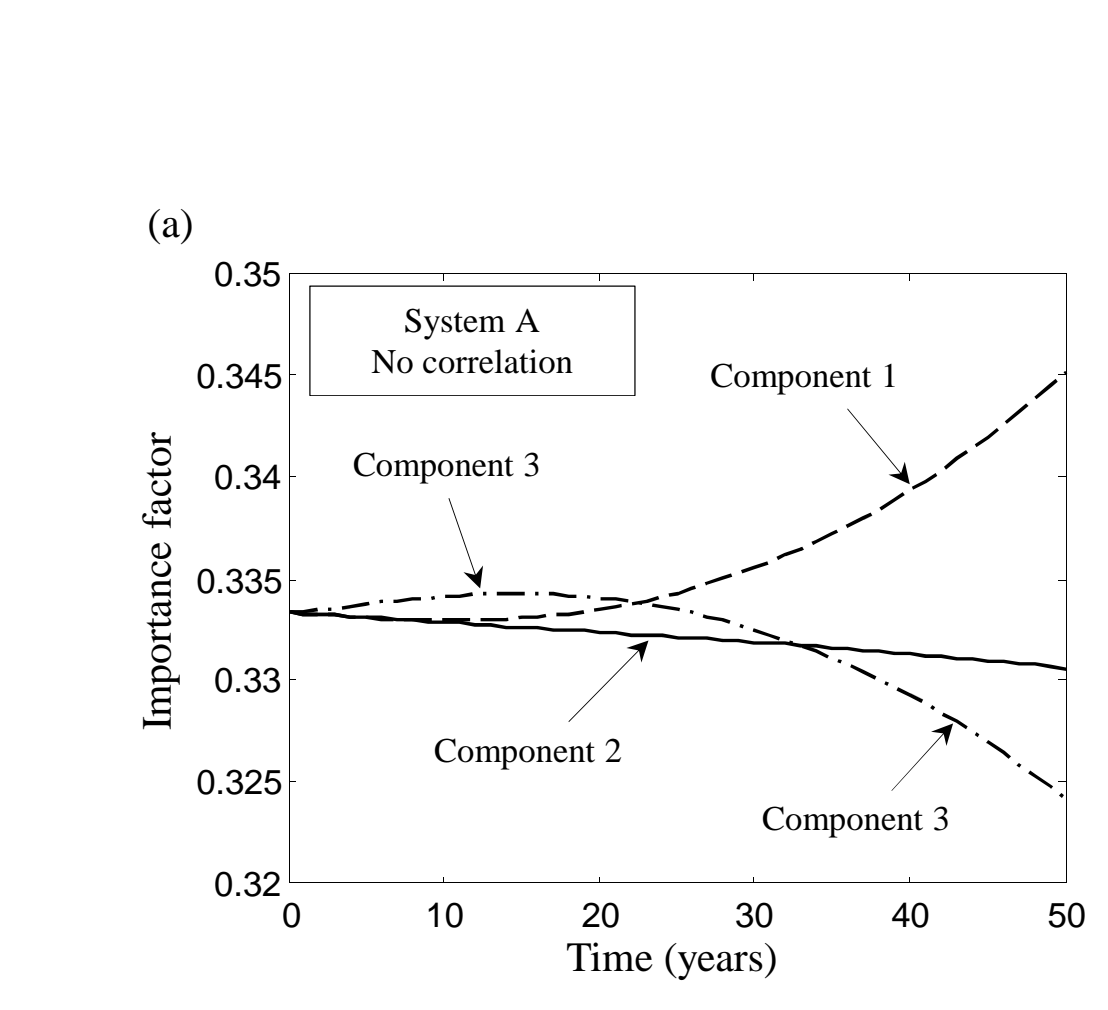

(c)

(b)

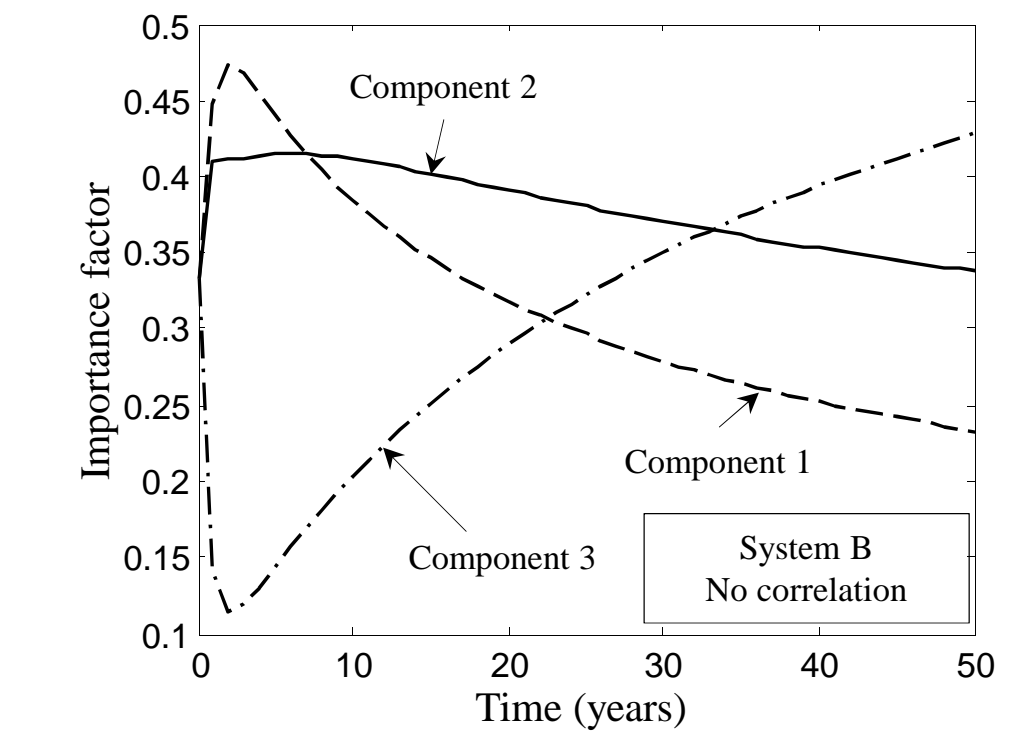

)

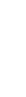

.




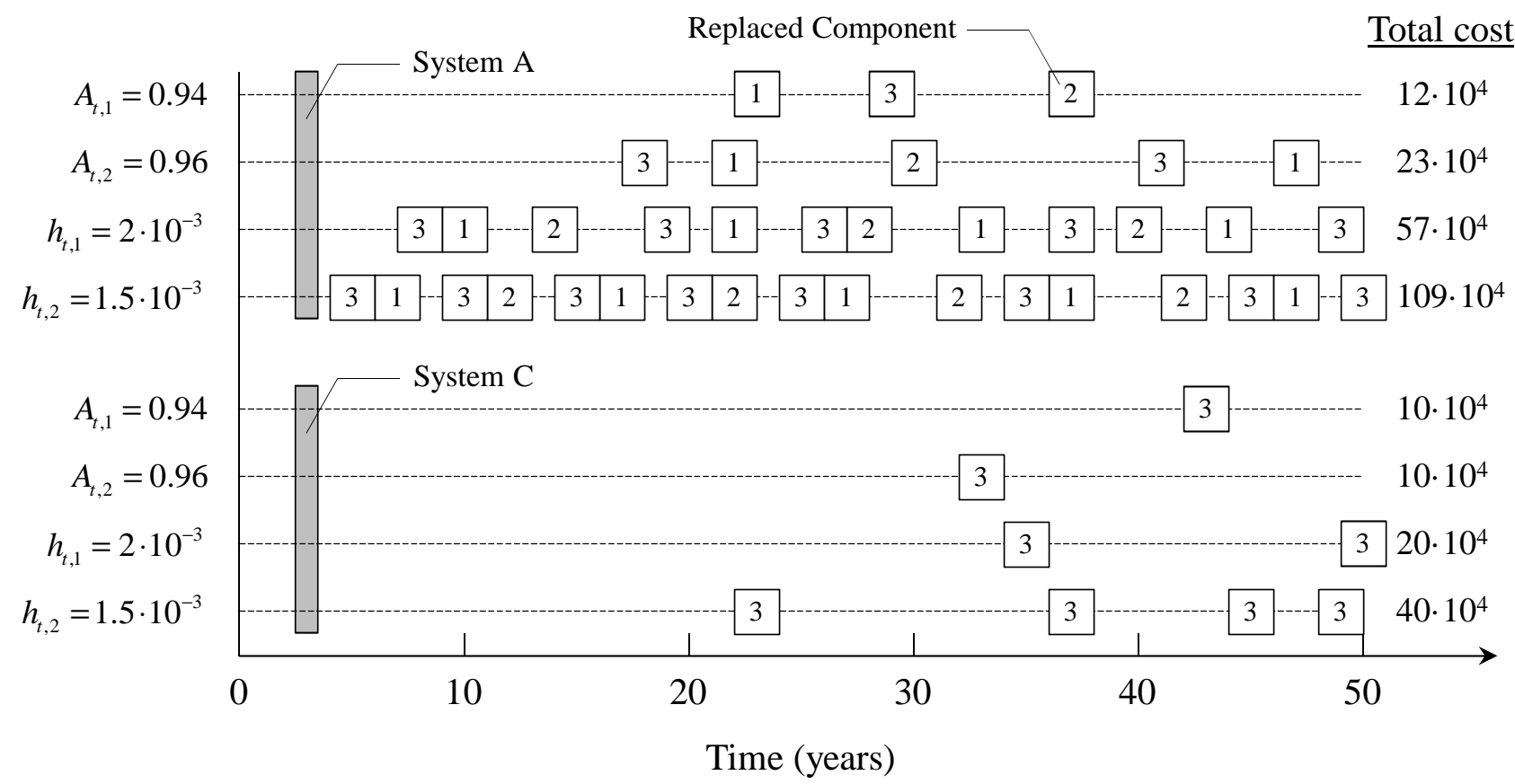

Total cos

$7 \cdot 10^{4}$

. $10^{4}$

$40 \cdot 10^{4}$

Time (years) 


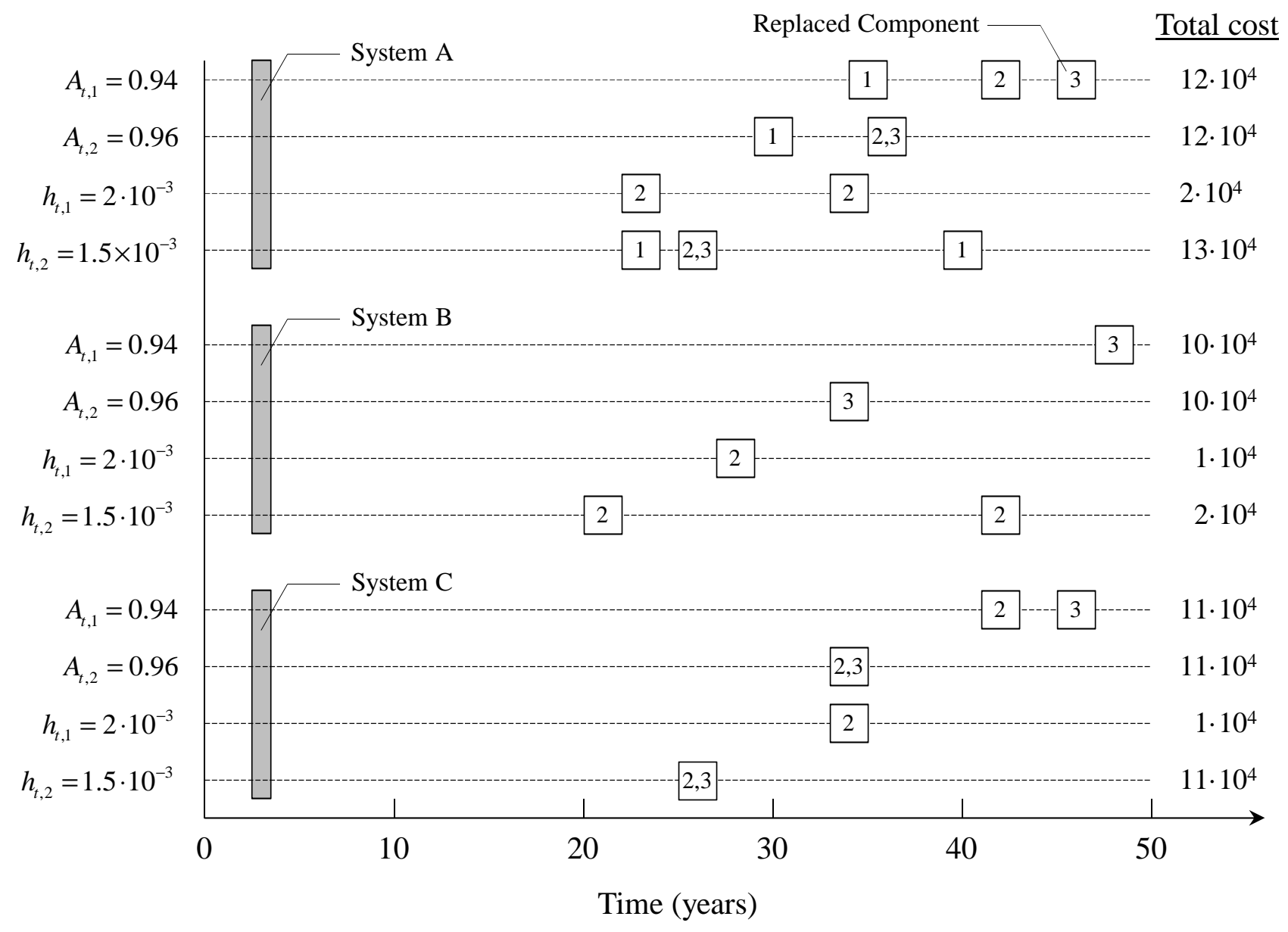


(a)

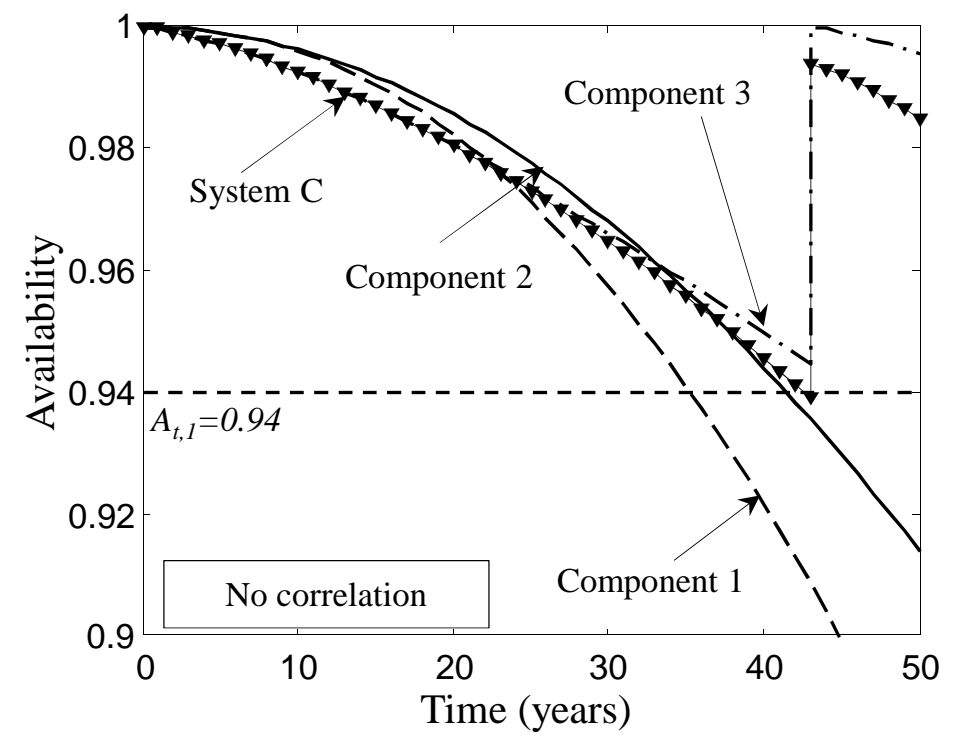

(c)

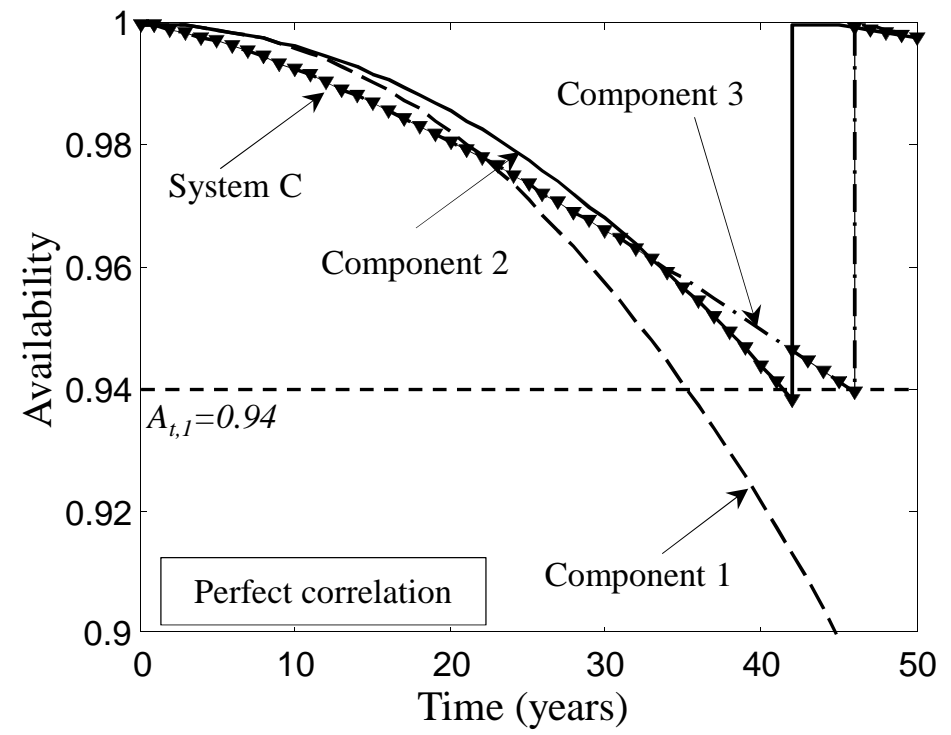

(b)

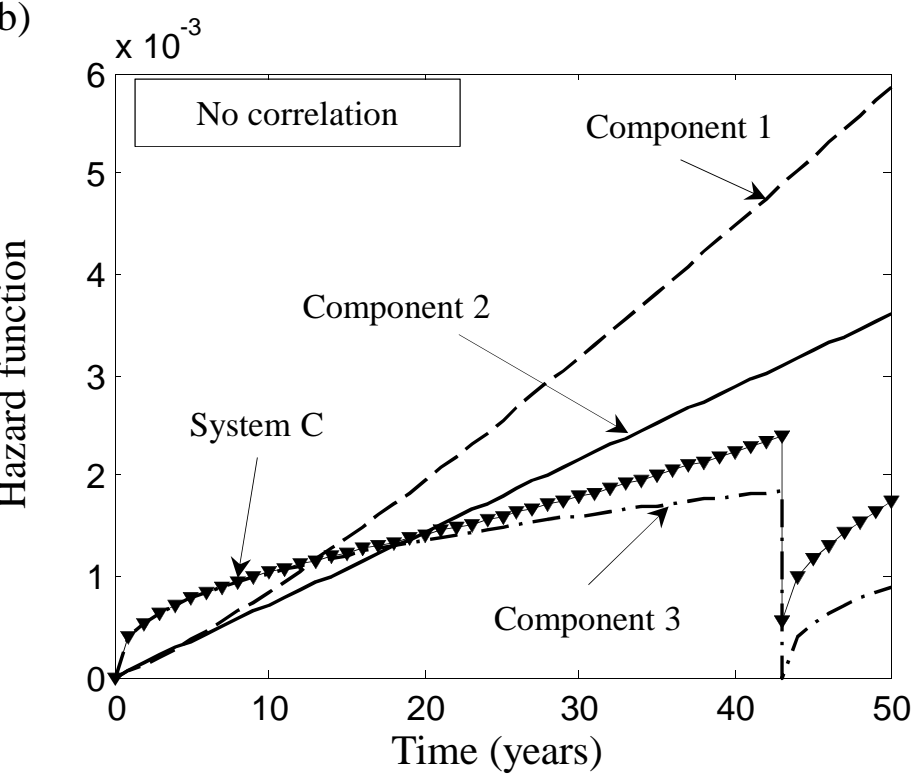

(d)

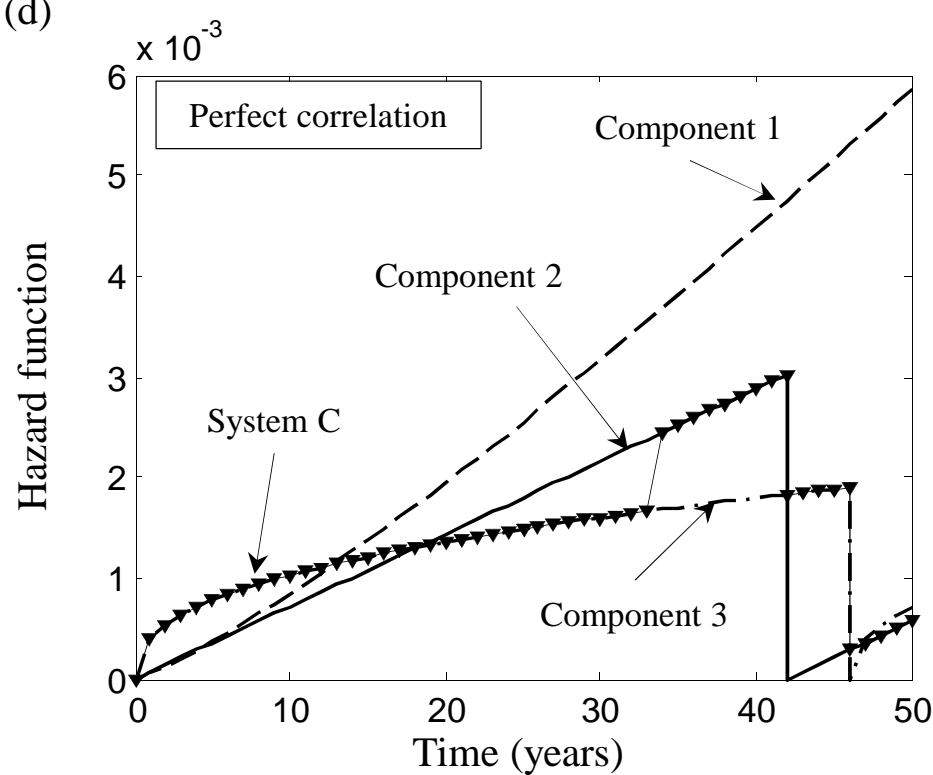



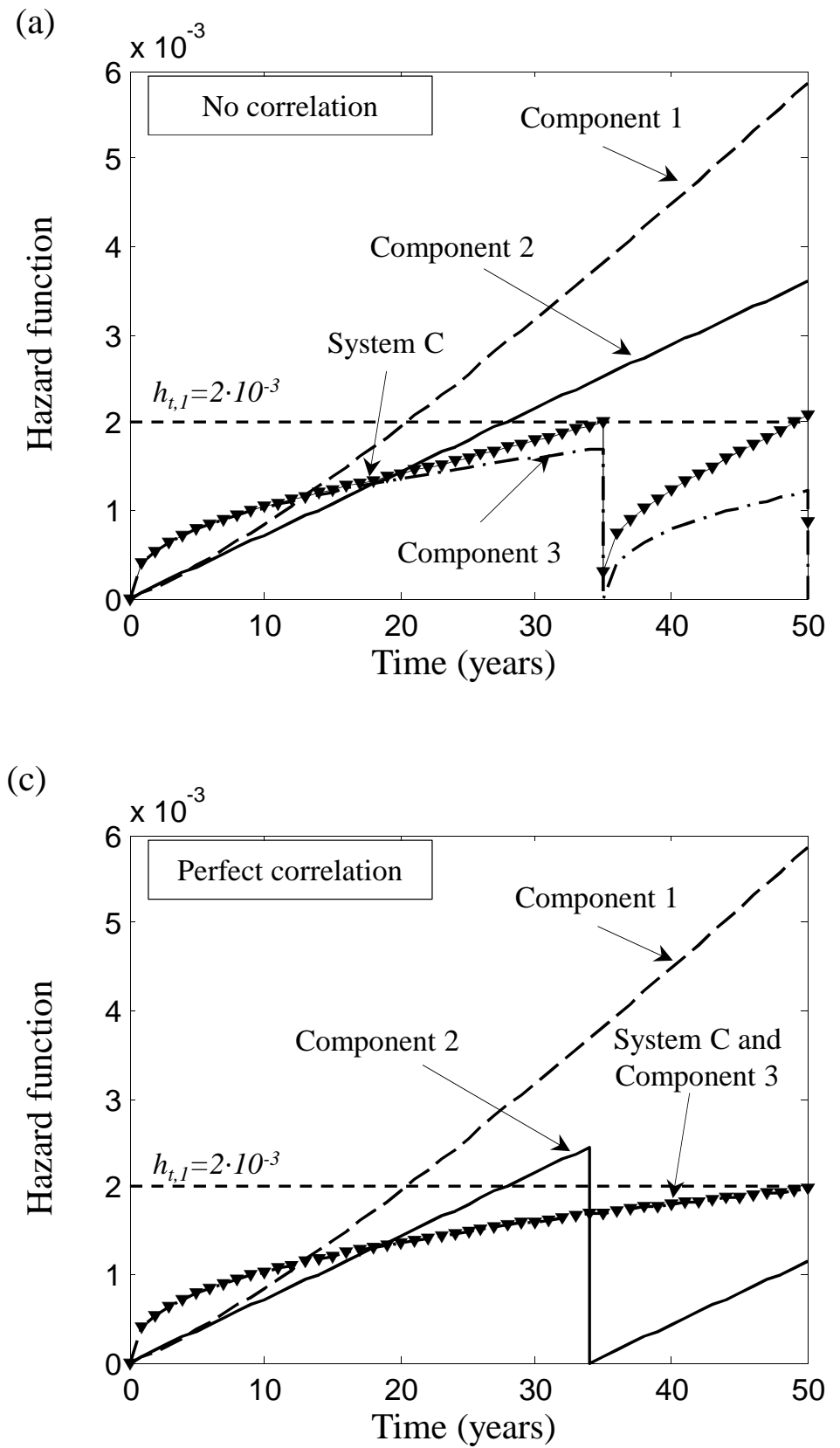
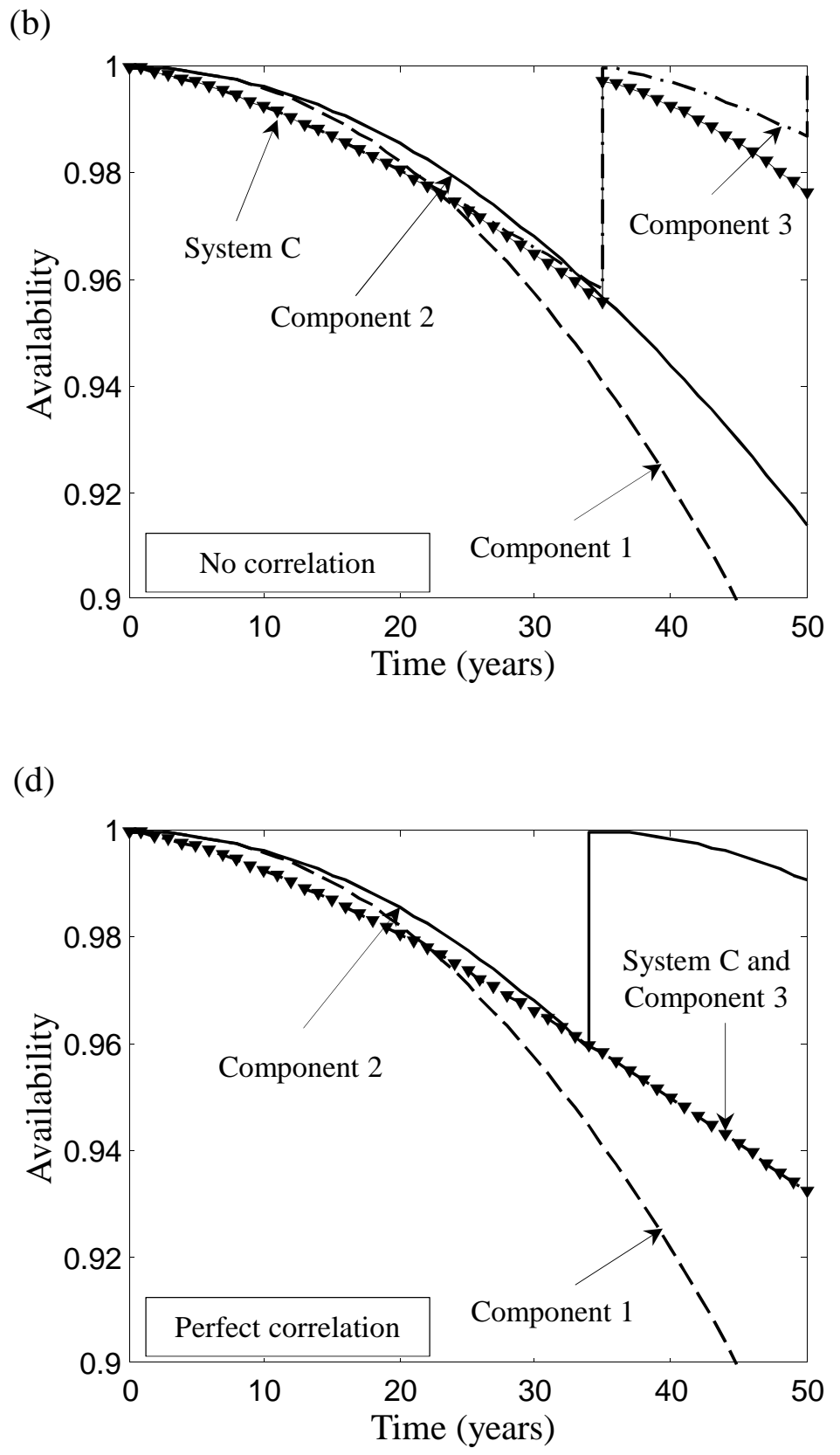
(a)

$9.144 \mathrm{~m}$

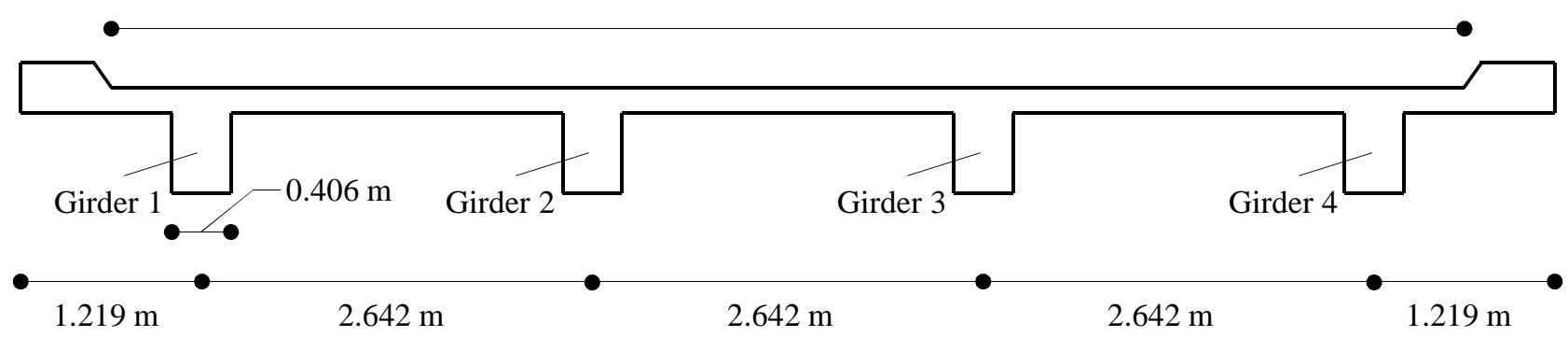

(b)

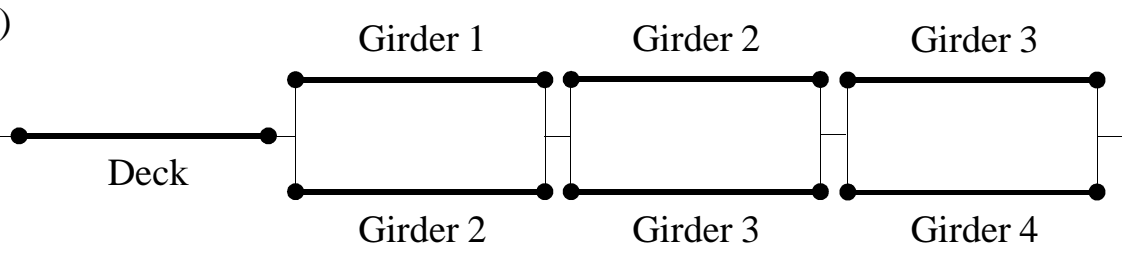


(a)

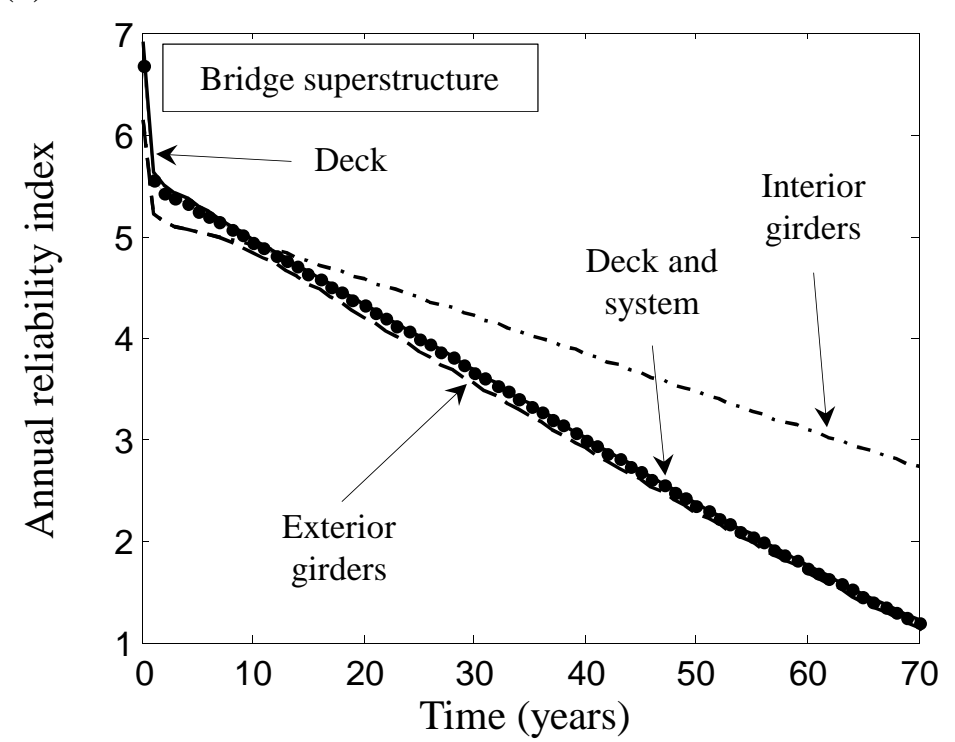

(b)

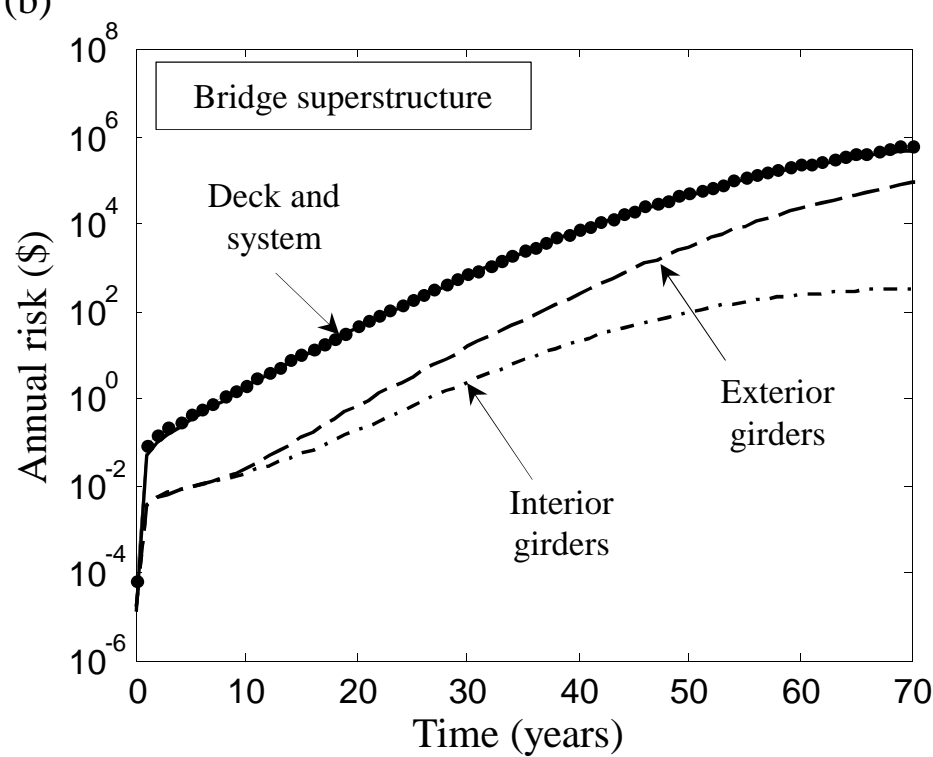


(a)

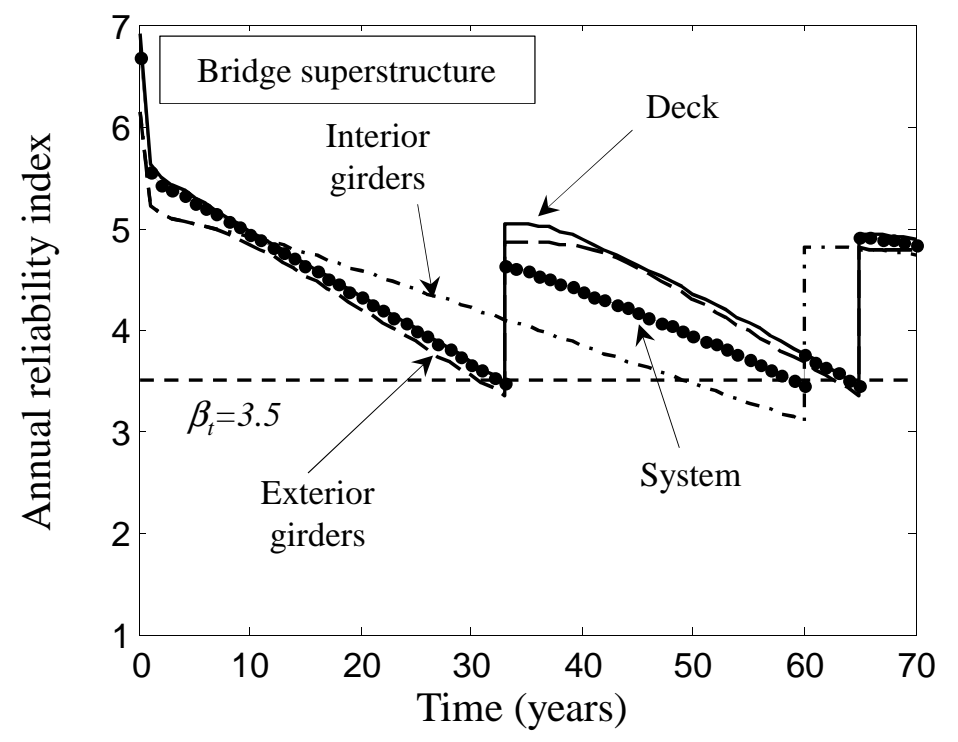

(c)

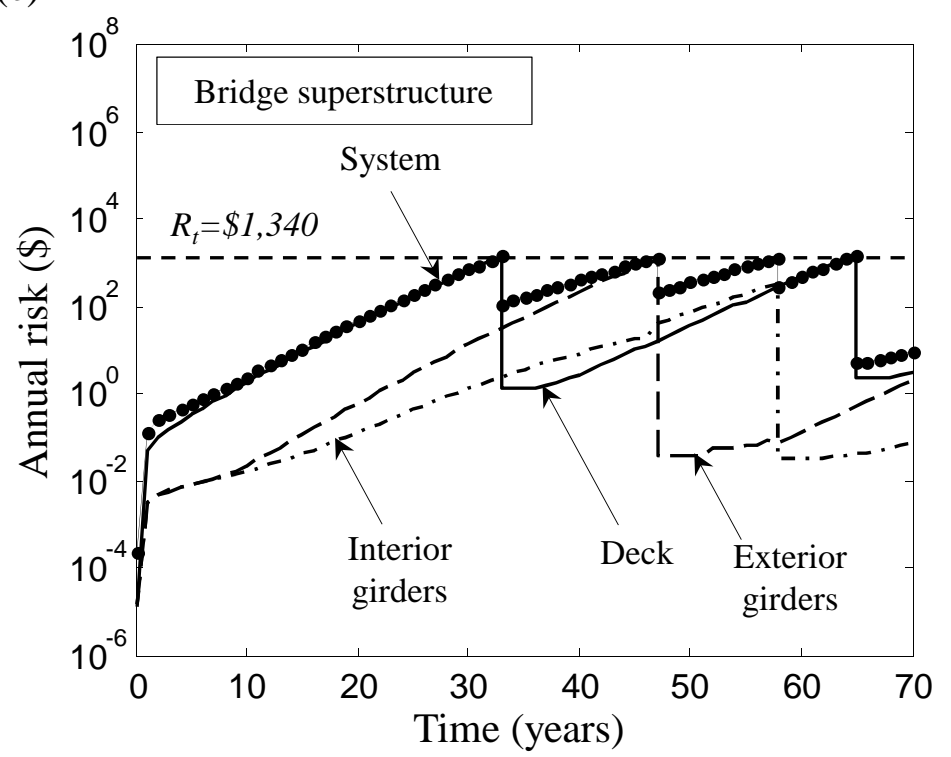

(b)

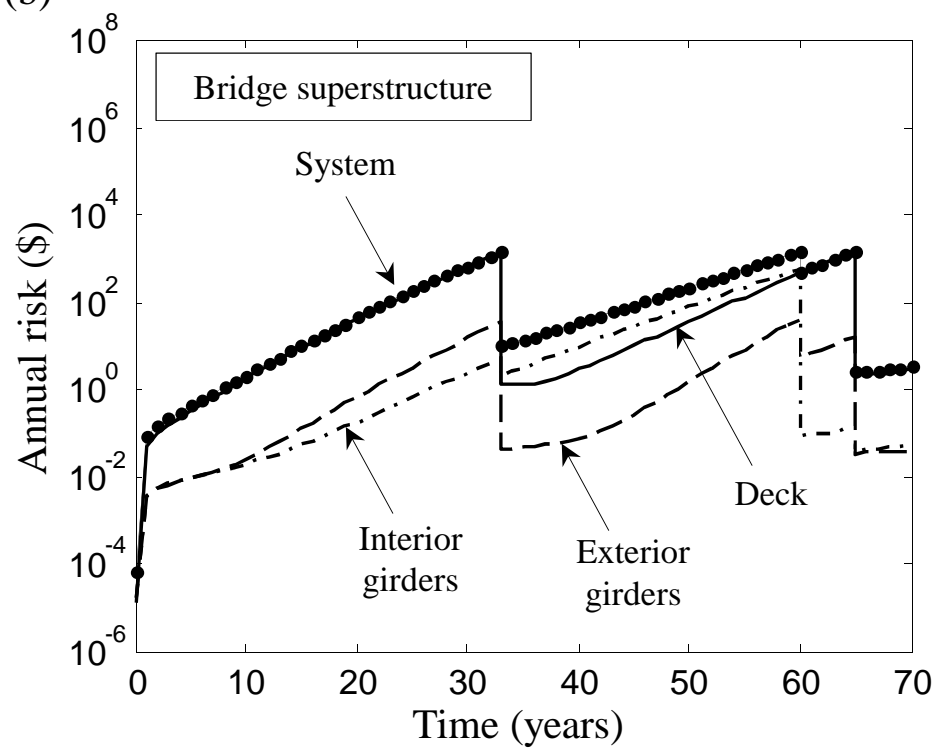

(d)

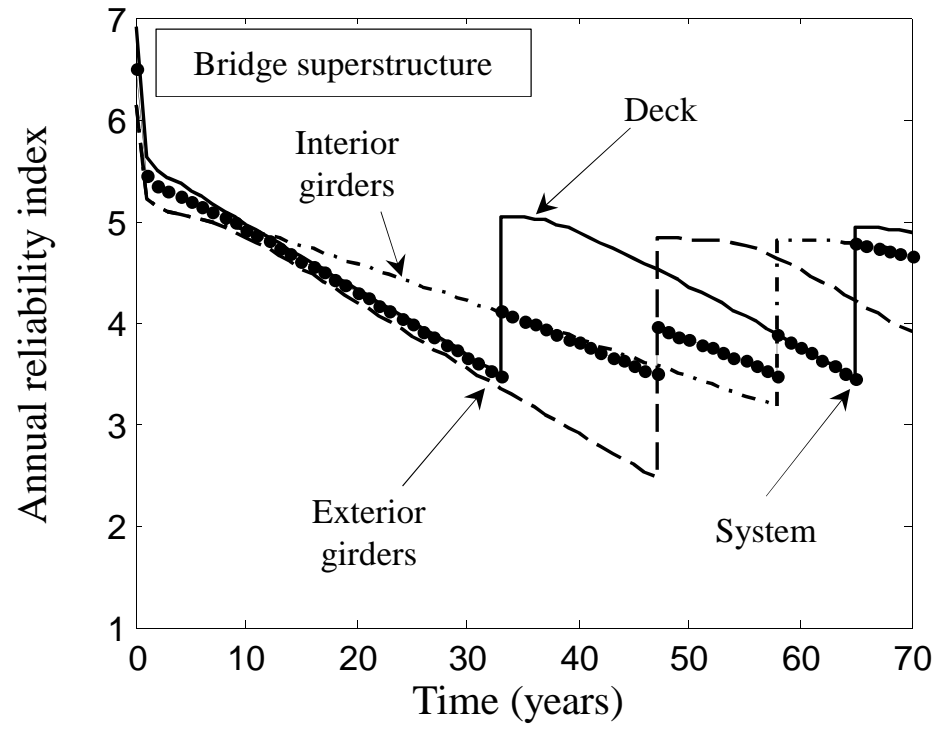


(a)

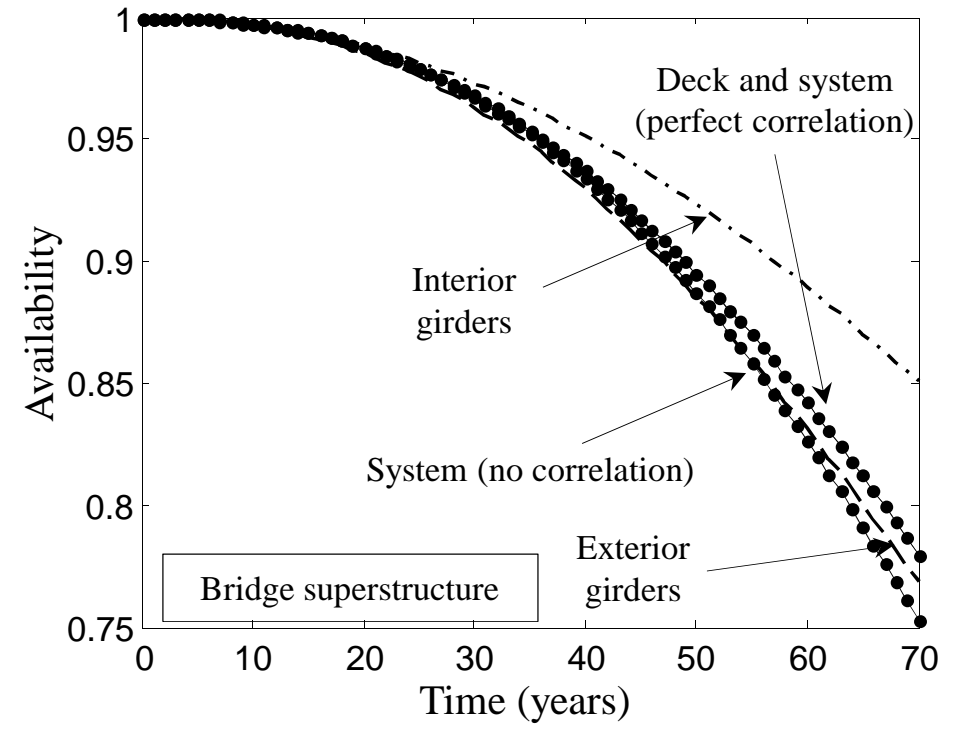

(b)

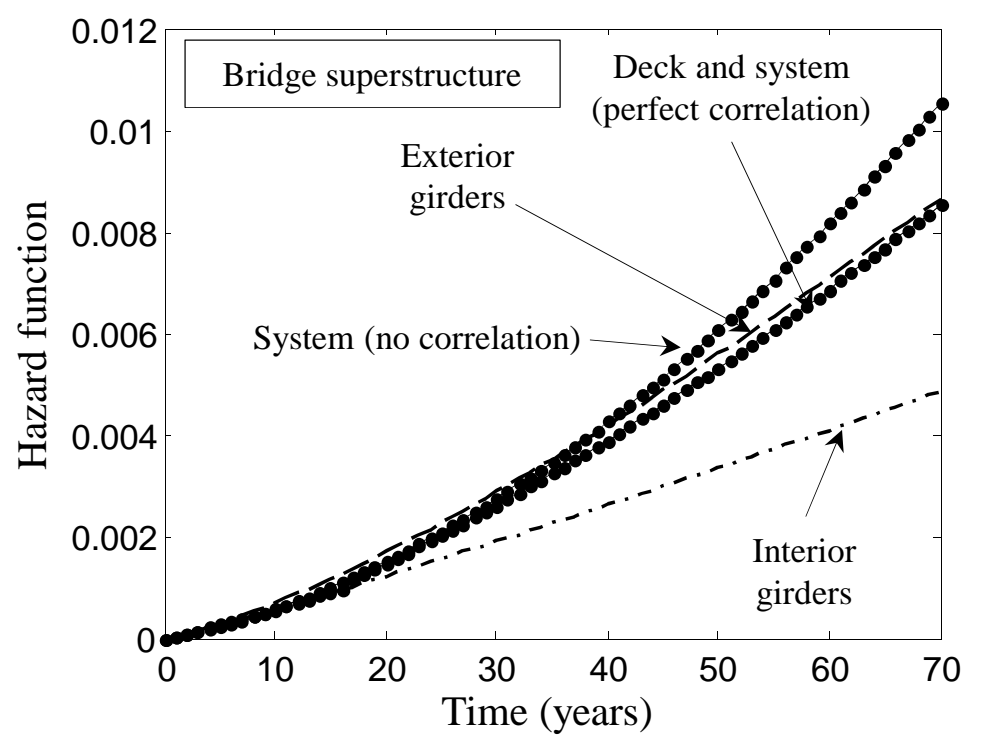


(a)

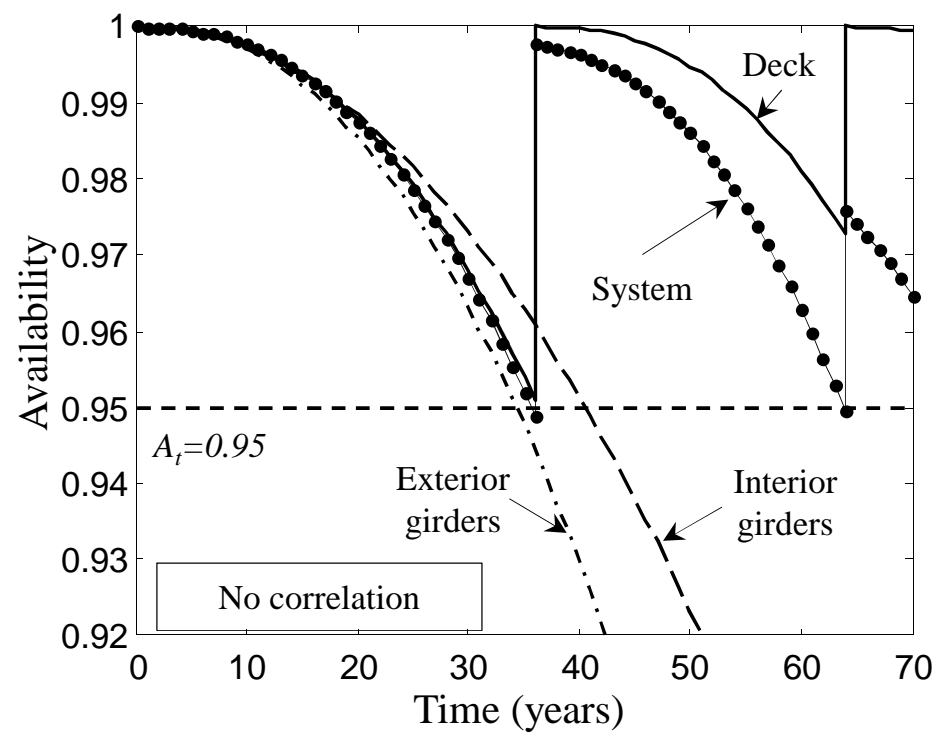

(c)

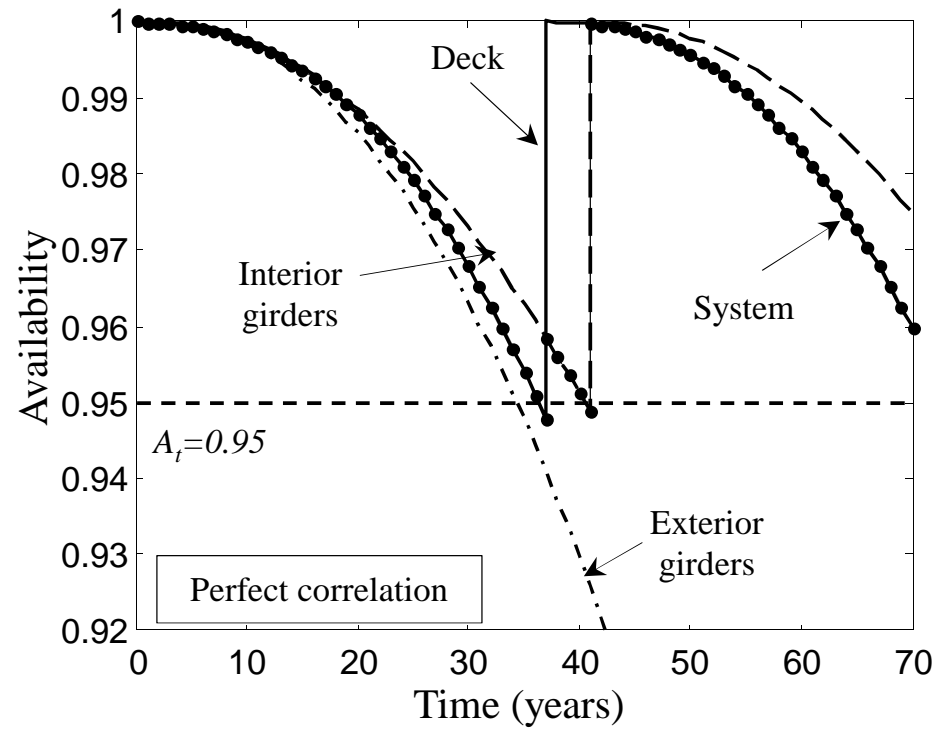

(b)

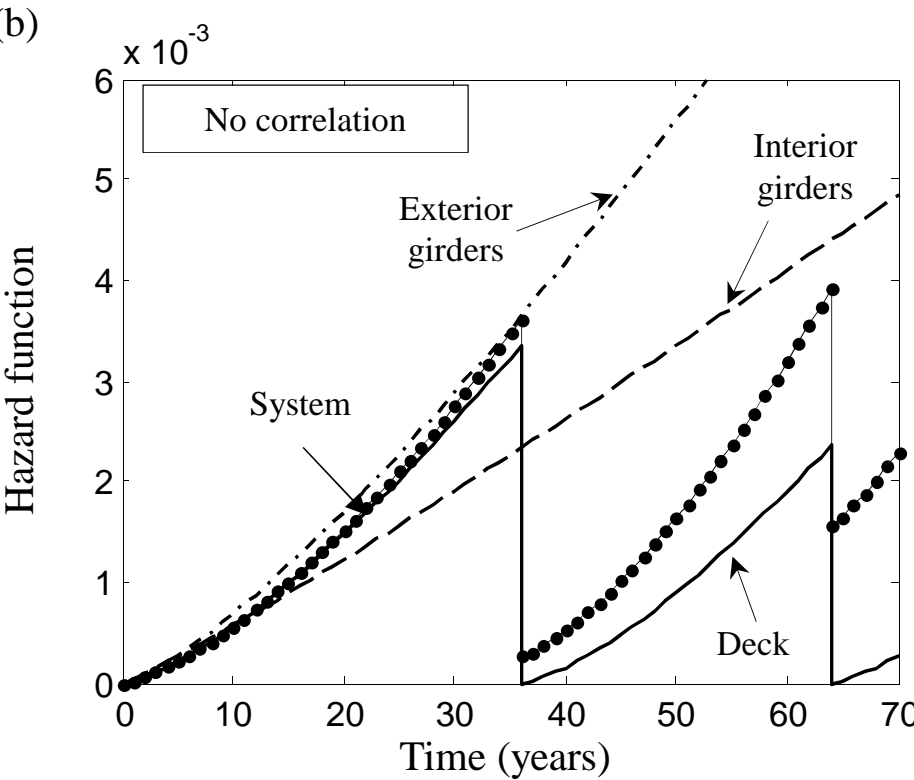

(d)

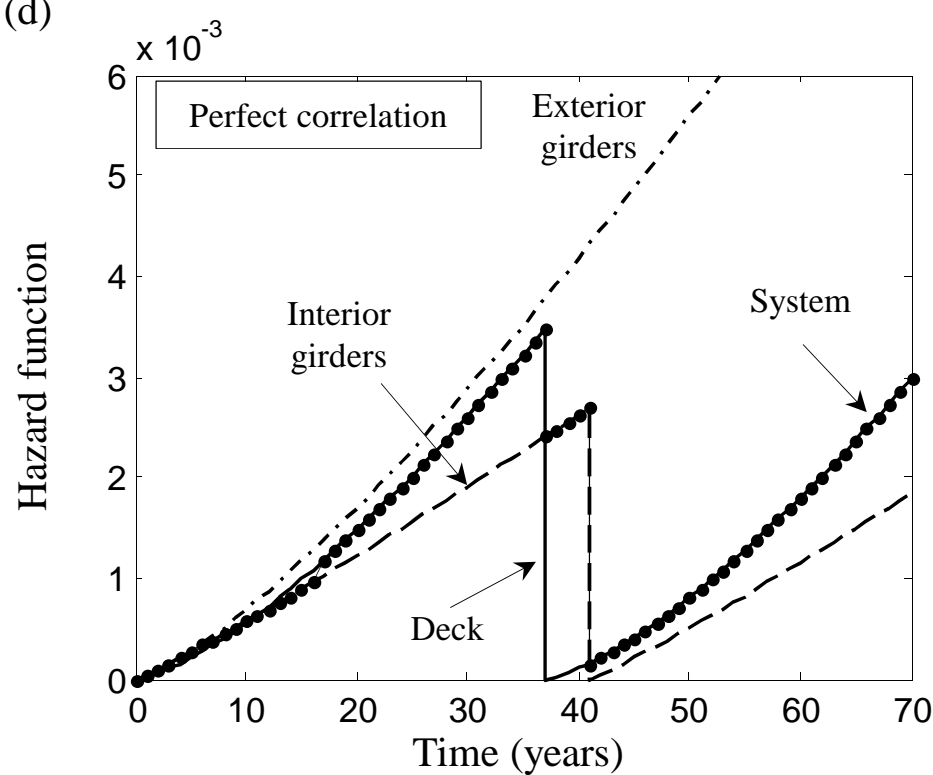


(a)

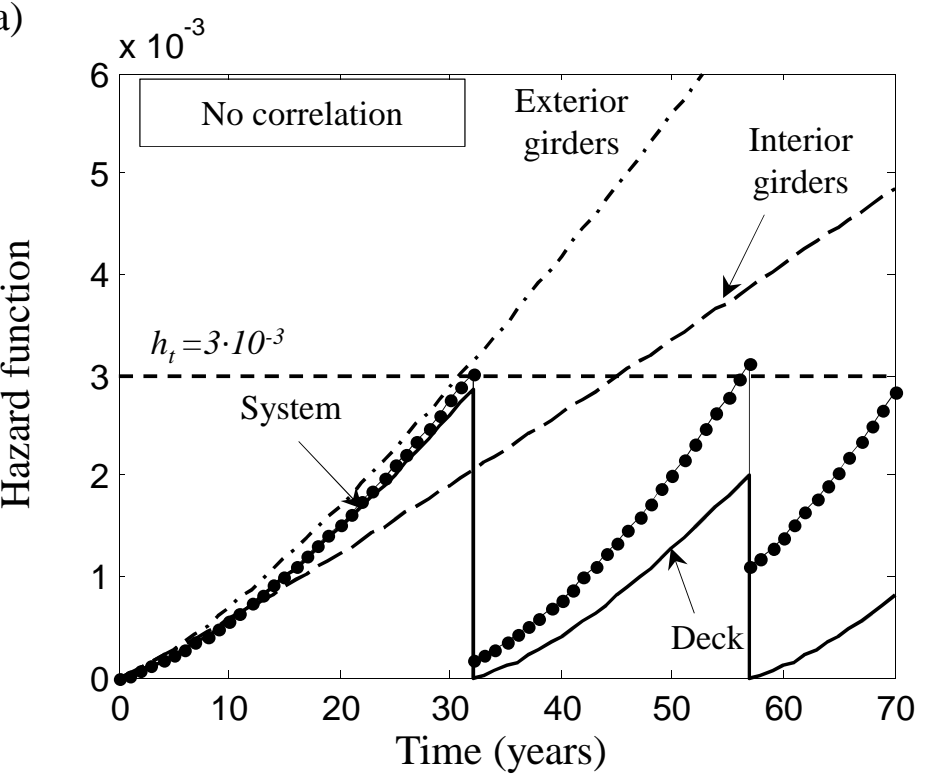

(c)

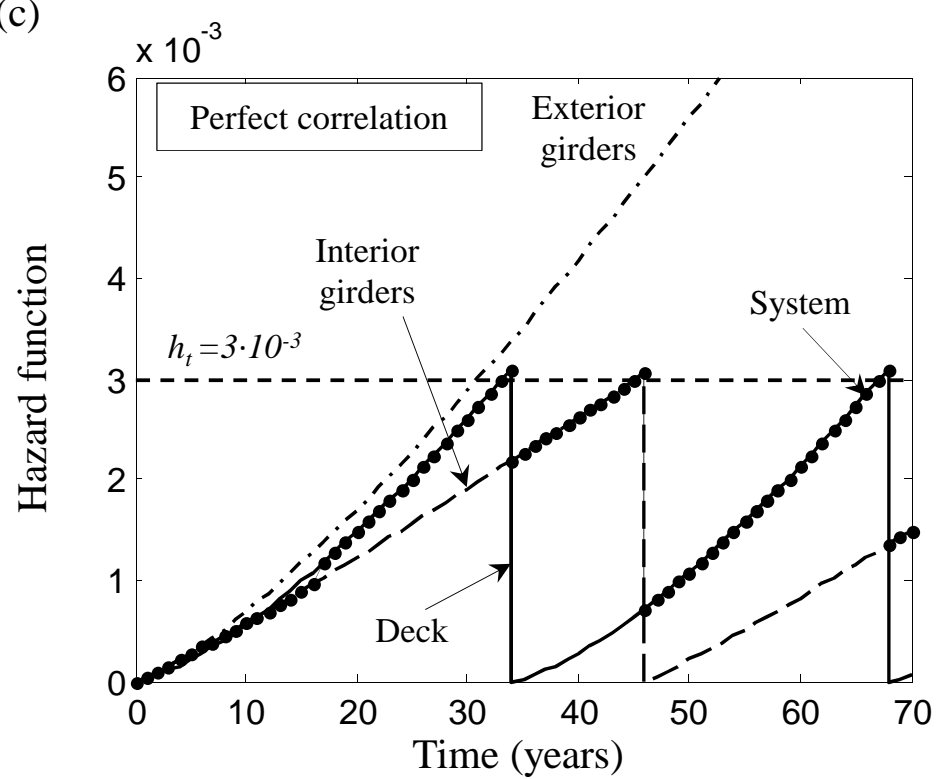

(b)

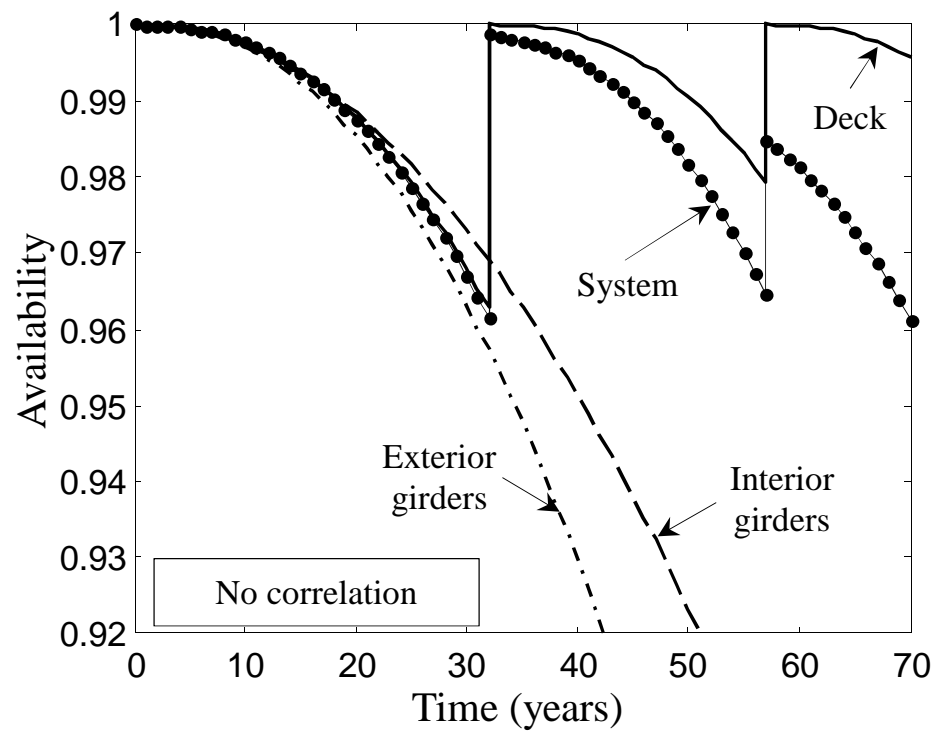

(d)

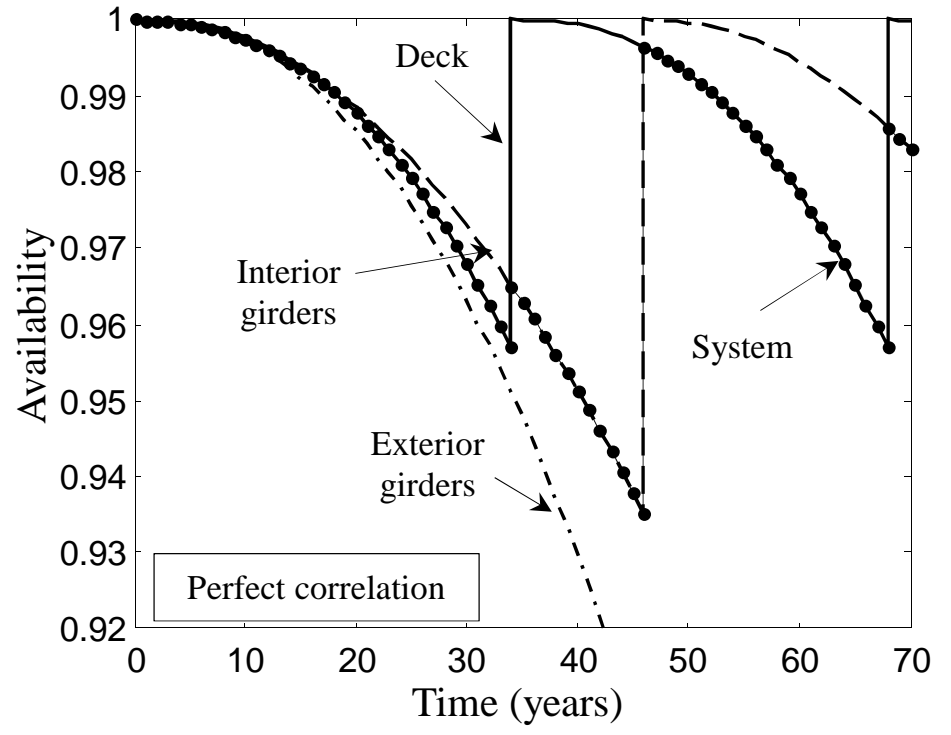

